\title{
ON THE LAW OF EFFECT ${ }^{1}$
}

\author{
R. J. Herrnstein
}

\author{
HARVARD UNIVERSITY
}

\begin{abstract}
Experiments on single, multiple, and concurrent schedules of reinforcement find various correlations between the rate of responding and the rate or magnitude of reinforcement. For concurrent schedules (i.e., simultaneous choice procedures), there is matching between the relative frequencies of responding and reinforcement; for multiple schedules (i.e., successive discrimination procedures), there are contrast effects between responding in each component and reinforcement in the others; and for single schedules, there are a host of increasing monotonic relations between the rate of responding and the rate of reinforcement. All these results, plus several others, can be accounted for by a coherent system of equations, the most general of which states that the absolute rate of any response is proportional to its associated relative reinforcement.
\end{abstract}

A review of the evidence for the law of effect would quickly reveal that the simple notion of "stamping-in" (Thorndike, 1911, e.g., p. 283) does not suffice. Animals do not just repeat the first successful act; they are likely to improve upon it until they find something like the optimal performance. In Thorndike's puzzle box, in the maze, or in Skinner's operant conditioning chamber, animals tend toward faster, easier, and more congenial movements, unless the performances are virtually optimal to begin with. Although some theorists find enough stereotypy to suggest a quasi-mechanical process of stamping-in (e.g., Guthrie and Horton, 1946), others have remained unconvinced (e.g., Tolman, 1948). Something more than the static form of the law of effect is needed for a really persuasive theory. The temptation to fall back on com-

\footnotetext{
${ }^{1}$ The preparation of this paper and the original work described herein were supported by grants to Harvard University from the National Science Foundation, the National Institute of Mental Health (NIMH-15494), and the National Institutes of Health (NIH-GM15258). This paper is dedicated to B. F. Skinner in his sixty-fifth year, in partial payment for an intellectual debt incurred over the past decade and a half. There are also more recent debts to be acknowledged, which I do gratefully. J. A. Nevin and H. S. Terrace have generously made available unpublished data, some of which have been included. W. M. Baum and J. R. Schneider have criticized and undoubtedly improved the paper, in substance and in style. Reprints may be obtained from the author, Psychological Laboratories, William James Hall, Harvard University, Cambridge, Massachusetts 02138.
}

mon sense and conclude that animals are adaptive, i.e., doing what profits them most, had best be resisted, for adaptation is at best a question, not an answer. And it is not hard to find evidence that violates both the Thorndikian principle of stamping-in and commonsense motions of adaptation, as the following two examples show.

Ferster and Skinner (1957) reported that an animal, when shifted from an interval to a ratio schedule, typically showed a change in its rate of responding. As regards stamping-in, the rate should remain unchanged for ratio schedules reinforce all rates of responding with equal probability (Morse, 1966). Although the deviation from the theory is large and reproducible, its direction is somewhat unpredictable. For example, in an experiment with pigeons (Ferster and Skinner, 1957, pp. 399407 ), one subject's rate of responding increased while the other's virtually ceased, when the schedule changed from a variable interval to a variable ratio matched for numbers of responses per reinforcement. While both of these findings-both the increase and the decrease in the rate of responding in the shift from interval to ratio schedule-violate the Thorndikian law of effect, only the increase is plausibly seen as adaptive. By responding faster on the ratio schedule, one animal increased its reinforcements per unit time, but, by the same token, the other one reduced its rate of reinforcement by responding more slowly. If the acceleration is adaptive, then the 
deceleration is not, and both findings are well substantiated.

A related finding, also violating both the Thorndikian law of effect and adaptiveness, has been obtained with the conjunctive schedule, schematically shown in Fig. 1. This graph plots on the coordinates of a cumulative record the region over which responses are unreinforced. For the conjunctive schedule, it is the entire plane minus the shaded area within the right angle. In other words, the conjunctive schedule reinforces the first response after the occurrence of a certain number of responses ( $\mathrm{n}$ on the figure) and the passage of a certain period of time $(t)$. The schedule is specified by its component members: fixed interval and fixed ratio in the present instance. The conjunctive schedule in Fig. 1 would be called $a$ "conjunctive fixed-interval $t$, fixed-ratio $n+1 . "$ In the simple fixed-interval schedule, rapid responding is implicitly penalized, for faster responding increases the work per reinforcement. In contrast, ratio schedules exact no such penalty for responding quickly, for the amount of work per reinforcement is held constant. In fact, ratio schedules may favor rapid responding by arranging a direct proportionality between the rate of responding and the rate of reinforcement. The conjunctive schedule concatenates these features of ratio

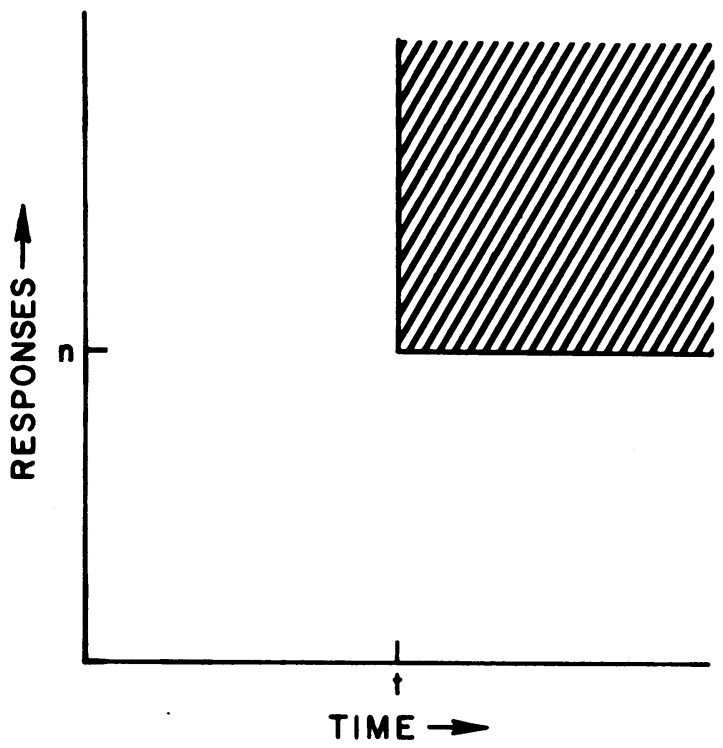

Fig. 1. The shaded area shows the region of reinforced responding on a conjunctive schedule of reinforcement. The ordinate is cumulated responding; the abscissa is elapsed time. and interval schedules, since the rate of reinforcement is directly proportional to the rate of responding only for rates of responding no larger than $n / t$, beyond which the rate of responding covaries with the responses per reinforcement.

The relevant finding was obtained in an experiment (Herrnstein and Morse, 1958) that held the interval component constant at 15 min, but varied the ratio component from zero (which is a simple fixed-interval schedule) to 240. Figure 2 shows the relation between rate of responding and the number requirement imposed by the ratio component. Although the pigeons were responding more than an average 300 times per reinforcement on the fixed-interval schedule, a number requirement as small as 10 (for one of the pigeons) or 40 (for either), caused a detectable slowing down of responding. The range of rates of responding within individual fixed intervals is large enough so that number requirements even this small are likely to make contact with the behavior. Larger requirements caused progressively larger decrements in responding. This falling rate of responding reduced the rate of reinforcement, as Fig. 3 shows. Here for the two pigeons are the average interreinforcement times as the number requirement was increased. For the fixedinterval schedule, the interreinforcement time was as small as the procedure permits, which is to say, $15 \mathrm{~min}$. Even the smaller requirements produced some reduction in the rate of reinforcement. For one pigeon, the rate of re-

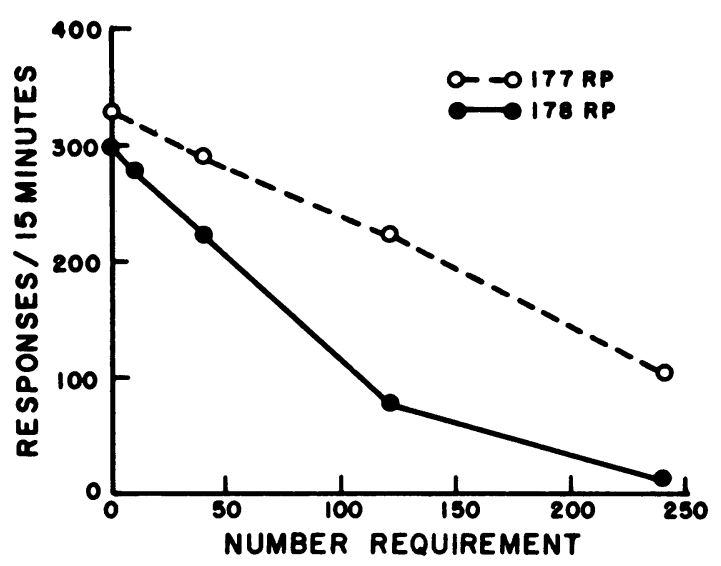

Fig. 2. For two subjects, the rate of responding as a function of the size of the number requirement in a conjunctive schedule with a time requirement of 15 min throughout. 


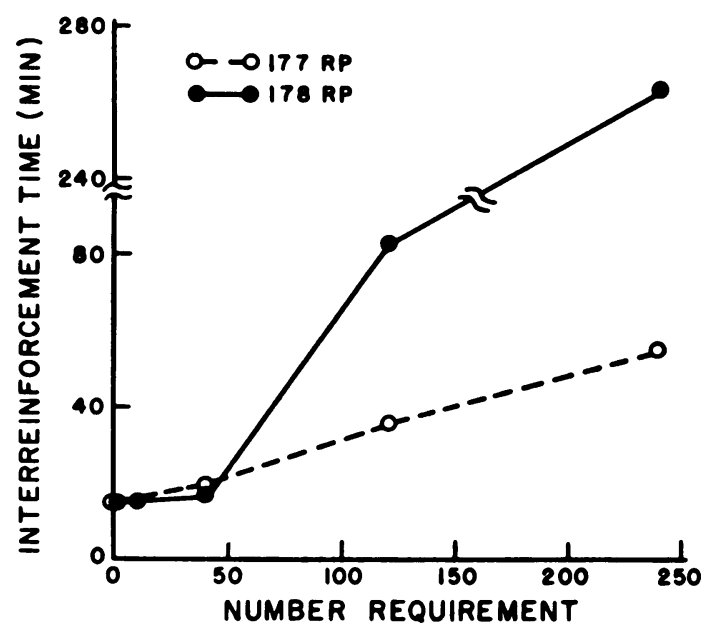

Fig. 3. For two subjects, the interreinforcement time as a function of the size of the number requirement in a conjunctive schedule with a time requirement of $15 \mathrm{~min}$ throughout.

inforcement fell very sharply as the number requirement was increased; for the other, the decline was more gradual, but in either case, the requirement took its toll throughout.

The conjunctive schedule lends itself poorly to a Thorndikian analysis, for what could be getting stamped-in when responding and response requirement vary inversely? Moreover, the conjunctive schedule makes poor sense as regards the animal's best interests, for the animal may be emitting substantially more (in Fig. 2, it was 30-fold more) behavior on the fixed interval than the number requirement demands, and yet the behavior is nevertheless depressed.

These problem cases are troublesome only within the confines of theory. In the broader sphere of common sense, reinforcement is affecting what might be termed the "strength" of behavior, as reflected in its rate. For example, consider the change from interval to ratio schedules. If the first effect is a higher rate of reinforcement, the rate of responding might increase. But this would further increase the rate of reinforcement, further "strengthening" responding, causing it to rise again, which again pushes the rate of reinforcement upwards, and so on. If, on the other hand, the first effect is a lower rate of reinforcement, then the rate of responding should fall. The rate of reinforcement would then also fall, further weakening the responding, and so on again. This dynamic process occurs with ratio, and not interval, schedules because only the ratio schedule arranges a proportionality between the rate of reinforcement and the rate of responding. The proportionality is the basis for an instability in ratio schedules that should produce either maximal responding or none at all, an implication that is confirmed by the tendency of ratio responding to be "two-valued" (Ferster and Skinner, 1957, chap. 4).

The conjunctive schedule similarly exemplifies the notion of strength. However slightly the number requirement increases the interreinforcement interval, it should reduce the strength, and therefore the output, of responding. If the strength of responding is sufficiently reduced so that the rate of responding is less than $n / t$. (see Fig. 1), then the conjunctive schedule becomes identical to a ratio schedule, and responding may reasonably be expected to vanish altogether, as it does with too-large ratios. One of the pigeons in Fig. 2 had, in fact, virtually stopped responding at the largest ratio studied, even though the number requirement was still less than the responses per reinforcement freely emitted on the simple fixed-interval schedule.

Those two examples, and others like them, show that neither stamping-in, nor adaptation, nor the two of them together, can account for what is here being called the strength of behavior. This paper specifies more formally than heretofore the shape of this intuitively obvious concept, while staying within the general outlines of the law of effect.

\section{REINFORCEMENT AS STRENGTHENING}

Reinforcement as strengthening is not being offered as a new idea, for "to reinforce" means to strengthen, and only by metaphor, to strengthen behavior. The earliest psychological usage concerned Pavlovian conditioning, where "reinforcement" of a reflex in a physiological sense was already familiar in classical work on facilitation and inhibition. The use of "reinforcement" in the vocabulary of instrumental learning was promoted in the mid1930s, particularly by Skinner and primarily as a substitute for the traditional term "reward", whose very age tainted it with the suspicion of mentalism. Mentalism notwithstanding, "reward" was more neutral than 
"reinforce", for while reward simply names a class of events that have some effect on the organism, "reinforcement" implies what the effect is, namely a strengthening. The extra connotation was tolerable only so long as it was not contrary to fact, which it was not. The leading advocates of the law of effect-Thorndike, Skinner, and others-had from the beginning spoken in terms of a "strengthening" of behavior.

What, though, does it mean to strengthen behavior? Thorndike's answer was the notion of stamping-in, which may, in fact, be adequate for the acquisition of new behavior. But for behavior already learned, stamping-in seems inappropriate. The response form as such is then no longer changing, and yet, as the examples in the previous section show, reinforcement is still affecting what might be considered the strength of the behavior. The answers of others, like Skinner and Hull, addressed themselves sensibly if not successfully to the underlying problem, which is one of measurement. To say that behavior is strengthened is to imply some dimension of behavior along which it changes when its strength changes.

The measurement problem is empirical, not conceptual, which is not to deny the virtue of clear and original thinking. It is, rather, to point out that the only persuasive argument for any measure of response strength is to show orderly relations between the parameters of reinforcement-its frequency, quantity, quality, and so on-and the designated parameter of behavior. The traditional measures of response-probability, rate, amplitude (i.e., work or effort), latency, resistance to extinction-have all failed to gain unequivocal support simply because orderly data with quantitative and general significance have not been forthcoming. Although there is no doubt that behavior is affected by its consequences, the law of effect is still expressed qualitatively, rather than as a relation between measurable variables, which it clearly must be at some level of analysis.

The notion of response probability comes closest to being a generally accepted measure of strength; cutting, as it does, across theories as diverse as those of Tolman (1938) and Hull (1943), Brunswik (1955) and Skinner (1953). But the agreement is more apparent than real, for the abstractness of "probability" masks the diversity of methods used for its extraction. For example, in some experiments, particularly those concerned with acquisition, the changing probabilities of response are estimated by the proportion of subjects doing something at successive points in training. In other experiments, single subjects are the basis for estimation of the probability by integrating over successive trials. In still others, the probability is estimated by the proportion of trials, or proportion of subjects, showing the choice of one response alternative out of a known set of alternatives. Not even the use of relative frequencies-the measure in modern probability theory-is common to all theorists, for according to Skinner, the rate of responding is the proper estimate of the organism's probability of responding. This is not an estimator in the formal sense-a mathematical probability is a dimensionless quantity between 0 and 1.0, and response rate is neither dimensionless nor bounded in principle-but rather an index of the animal's disposition to respond over some interval of time. Given the present state of knowledge, this abundance of measures is more likely to confuse than to enrich.

To reduce the confusion, and hopefully to advance the state of knowledge, the present approach focuses initially on a single relativefrequency measure as its index of strength. No "probability" will be inferred simply because to do so might suggest an equivalence with other empirical measures for which there is no evidence. The measure is exemplified by an experiment in which pigeons had two keys to peck (Herrnstein, 1961). The keys were available continuously during experimental sessions and pecking was reinforced with two variable-interval schedules, mutually independent and running simultaneously. The relative frequency is obtained by dividing the number of pecks on one key by the sum to both. In the context of operant conditioning this is a concurrent schedule, but it is clearly a version of the familiar "choice" experiment. It is, however, different in two significant respects. First, it uses continuous exposure to the alternatives instead of discrete trials. Second, reinforcements come on interval, instead of ratio, schedules. In the typical choice experiment, as well as in gambling casinos, the over-all probability of winning is constant for a given play (or response alternative), so 
that the number of wins is proportional to the number of plays. With interval schedules, there is no such proportionality, as noted earlier. Instead, the probability of winning on any given play is inversely related to the rate of play (response), and the number of wins is virtually independent of the number of plays, given a high enough rate of play.

The pigeons, then, had a pair of keys to peck, and the experiment rewarded their efforts with brief access to food at irregular intervals. The schedules set a maximum rate of reinforcement throughout the experiment at 40 per hour, but the number allocated to one key or the other was systematically varied, to see how the distribution of responses was affected. The question was whether, to use the vocabulary of this paper, response strength as relative frequency was some plausible function of reinforcement frequency. The answer was both plausible and attractively simple, as shown in Fig. 4. The ordinate is the proportion of responses on the left key; the abscissa is the proportion of reinforcements delivered thereon. The points fall near the diagonal, which is the locus of perfect matching between the distribution of responses and of reinforcements. A simple equation summarizes the finding ( $P$ is number of pecks, $R$ is number

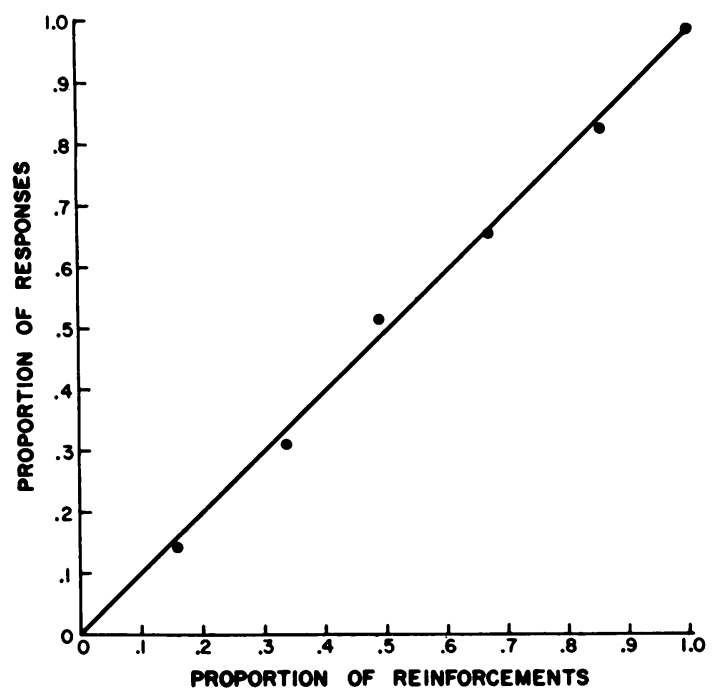

Fig. 4. The relative frequency of responding to one alternative in a two-choice procedure as a function of the relative frequency of reinforcement thereon. Variable-interval schedules governed reinforcements for both alternatives. The diagonal line shows matching between the relative frequencies. From Herrnstein (1961). of reinforcements, and the subscripts denote the two alternatives).

$$
\frac{\mathbf{P}_{\mathbf{L}}}{\mathbf{P}_{\mathbf{L}}+\mathbf{P}_{\mathbf{R}}}=\frac{\mathbf{R}_{\mathbf{L}}}{\mathbf{R}_{\mathbf{L}}+\mathbf{R}_{\mathbf{R}}}
$$

Unless the number of pecks far exceeds the number of reinforcements, the matching function (equation 1) is trivially true. For example, if the variable-interval schedules were assigning reinforcements to the two keys more quickly than the pigeons pecked, then every peck would be reinforced. The data point would necessarily fall on the diagonal, but the result would have little empirical content. If reinforcements were being assigned at the rate of one for every other response, or one for every third response, the possible range of variation for the points would still be narrowly constrained around the diagonal, and the findings would still be essentially vacuous. In fact, the possible range of variation is exactly fixed by the actual numbers of reinforcements and responses. Since there are at least as many responses on each key as there are reinforcements, the smallest relative frequency of response for a given relative frequency of reinforcement $R_{L} /\left(R_{L}+R_{R}\right)$ is

$$
\frac{\mathbf{R}_{\mathbf{L}}}{\mathbf{P}_{\mathbf{L}}+\mathbf{P}_{\mathbf{R}}}
$$

This fraction approaches $R_{L} /\left(R_{L}+R_{R}\right)$ as the total number of responses approaches the total number of reinforcements. The largest relative frequency of response for a given relative frequency of reinforcement is also dependent upon the fact that there can be no fewer responses than reinforcements. Thus, the ratio of responses for a given $R_{L} /\left(R_{L}+R_{R}\right)$ can go no higher than 1.0 minus the minimum value for the other key- $\mathbf{R}_{\mathbf{R}} / \mathbf{P}_{\mathbf{L}}+\mathbf{P}_{\mathbf{R}}$ )-which may be written as

$$
\frac{\mathbf{P}_{\mathbf{L}}+\mathbf{P}_{\mathbf{R}}-\mathbf{R}_{\mathbf{R}}}{\mathbf{P}_{\mathbf{L}}+\mathbf{P}_{\mathbf{R}}}
$$

This fraction, too, will approach $\mathbf{R}_{\mathrm{L}} /\left(\mathbf{R}_{\mathbf{L}}+\mathbf{R}_{\mathbf{R}}\right)$ as the total number of responses approaches the total number of reinforcements, which is to say when the responses on the separate keys each equal the reinforcements thereon. The result, then, is that as the number of responses approaches the number of reinforcements, the possible range of variation for the ratio of responses converges on to the matching relation in Fig. 4. In the case of the experiment sum- 
marized here, however, the ratio of responses to reinforcements was approximately 100 . At 0.5 on the abscissa, therefore, the possible range of variation of responding was from 0.005 to 0.995 . At other values of the abscissa, the possible range was comparably broad. The close agreement between the matching relation and the distribution of responding says something, therefore, about the animal, not just about the procedure itself. What this is, and how it operates in a variety of situations, will occupy the remainder of the paper.

\section{RESPONSE STRENGTH AND CHOICE}

The experiment summarized in Fig. 4 had an additional procedural wrinkle. Each time the pigeon shifted from one key to the other, there was a brief period of time during which any reinforcement called for by the variableinterval schedule was delayed. This "changeover delay" (COD) lasted for $1.5 \mathrm{sec}$, and was imposed in order to prevent the pigeons from switching after virtually every response. It was found, without the COD, that the distribution of responses tended to stay around 50-50, without regard to the distribution of reinforcements. If the matching relation were an accident of the duration of the COD, it would hardly be a principle of either response strength or choice. The most direct test of the COD is contained in an experiment in which it was varied systematically to see whether the matching relation was a peculiarity of only a certain range of durations.

The experiment, by Shull and Pliskoff (1967), varied a number of conditions besides the duration of the COD. Instead of pigeons, their experiment used albino rats. The reinforcer, instead of brief access to food for a hungry animal, was the opportunity for the rat to get electric current passed through its brain in the region of the posterior hypothalamus. A reinforcement consisted of the lighting of a small light in the presence of which the rat could press the lever 20 times, each time obtaining 125 milliseconds of a $100-\mathrm{Hz}$ train of sine waves of 150 to 300 microamps across the electrodes implanted in its head. The variable-interval schedule resumed at the end of the last burst of current. The schedule itself was a variation of the simple concurrent procedure, one that had been originally described by Findley (1958). Instead of a pair of response alternatives associated with a pair of variableinterval schedules, the Findley procedure had the two variable intervals associated with a pair of stimuli, but responses on only one of the two levers could produce reinforcement. At any one time, only one of the two stimuli was present, and while it was present, reinforcements from only its associated variable interval were forthcoming. The second lever in the chamber switched from one stimulus to the other, along with its associated variableinterval schedule. Actually, the two variableinterval programmers ran concurrently and continuously, just as they do in a conventional concurrent procedure. Shull and Pliskoff varied the COD, which is here the minimum possible interval between a response to the switching lever and the first reinforced response. Their finding was that matching occurred as long as the COD was greater than a certain minimal duration, as was found in the earlier study, but that beyond that value, matching was maintained whatever the duration of the COD in the range examined (0 to $20 \mathrm{sec}$ ). As the COD is made larger, however, it begins to affect measurably the obtained rates of reinforcement by interacting with the schedules themselves, as might be expected. Matching is always with respect to obtained, rather than pre-specified, reinforcement rates.

The experiment by Shull and Pliskoff extended the generality of the matching relation more than merely by showing that the COD is not the controlling variable. It extended the finding to rats, to Findley's procedure, and to intracranial stimulation as the reinforcer. Other studies have extended it further. Reynolds (1963a) showed matching with three response alternatives instead of two. Holz (1968) found matching even when each of the responses was punished with electric shock, in addition to being reinforced by the usual variable-interval schedules. Holz varied the intensity of the punishment until it was so severe that the pigeons stopped responding altogether. However, as long as they were responding, and as long as the punishment for the two responses was equally intense, the distribution of responses matched the distribution of reinforcements. Catania (1963a) and Neuringer (1967b) found matching with respect to total amount of food when the two reinforcers differed not in their rate of occur- 
rence, but in the grams of food per reinforcement. In another study, Catania (1963b), using the Findley procedure, found matching both for the proportion of responses and for proportion of time spent with each of the two stimuli. Baum and Rachlin (1969) showed matching (correcting for a position bias) when the "responses" consisted of standing on one side of a chamber or the other. The proportion of time spent in each location was found to be distributed as the associated proportion of reinforcements. Along these lines, Brownstein and Pliskoff (1968) found that the Findley procedure can be further modified so that when the animal selects one stimulus condition or the other, reinforcement comes along independently of any response. The pigeons here are simply choosing between one rate of reinforcement or the other. Their finding, too, is described by the matching relation, suitably adapted. The proportion of time spent in each stimulus condition is equal to the proportion of reinforcement received therefrom. Nevin (1969) noted that matching is found in human psychophysical studies when the proportion of "yes" responses is plotted against either the proportion of trials containing a signal or the relative size of payoff (i.e., the frequency or magnitude of reinforcement). Shimp (1966), and the present author in unpublished work, have found matching in discrete-trial procedures of various sorts.

The list of confirmatory studies could be extended, but without profit at this point. It has been consistently found that responding is distributed in proportion to the distribution of reinforcement, as long as the responding and the reinforcements across the alternatives are not unequal qualitatively. Thus, the matching relation would not be expected if the reinforcer for one response were a preferred food and that for the other were a nonpreferred food, unless the scale values for the reinforcers expressed the difference quantitatively. Nor would it be expected if the two responses differed in some important way, e.g., that one involved considerably more work than the other. In fact, the matching relation may be used to construct equivalences between qualitatively different responses or reinforcers, although no such undertaking has come to the author's attention. It should, however, be possible to scale reinforcers against each other or responses against each other by assuming that the subject must be conforming to the matching relation whenever it is in a choice situation of the general type employed in these experiments, and by adjusting the measures of response or reinforcement accordingly.

The main opposition to the matching relation is found in the literature on so-called "probability learning". If an experiment arranges a certain probability (excluding 1.0, 0.5 , and 0 ) of reinforcement for each of a pair of response alternatives, and if the subject distributes its responses in proportion to these pre-assigned probabilities, then the matching relation, as defined here, is violated. Imagine that the two probabilities are 0.4 and 0.1 . In a sequence of 100 responses, probability learning requires 80 responses to the better alternative and 20 responses to the poorer one. The number of reinforcements would be $80 \times 0.4=32$ for the one, and $20 \times 0.1=2$ for the other. With respect to the matching formula, this is a violation, for

$$
\frac{80}{80+20} \neq \frac{32}{32+2}
$$

The literature does not, however, claim strict conformity to probability learning. Instead, responding is often confined exclusively to one or the other of the two alternatives, typically toward the alternative with the higher probability of reinforcement. But even when the two reinforcement probabilities are equal, responding tends to become exclusive for one of the choices. These deviations from probability learning are said to be instances of "optimizing" or "maximizing", since exclusive preference is the optimal strategy in the sense that the subject will, on the average, maximize its winning if it stays with the better bet. Even when the two probabilities are equal, nothing is lost by exclusive preference, and perhaps something is gained, for the subject is thereby spared the effort of switching from one alternative to the other.

Maximization in the probability type experiment actually conforms to the present matching function. Equation ( 1 ) is satisfied when all or none of the responses and all or none of the reinforcements occur for one of the alternatives and is therefore consistent with all of the experiments that deviate from probability learning and find maximization instead. Not all the experiments, however, find 
exclusive preference, and it is not yet clear whether apparently minor procedural factors are inadvertently affecting outcomes, as some have suggested (Bush and Mosteller, 1955), or whether there is, in addition, a phyletic factor. Bitterman (1965) argued that the higher organisms tend to maximize, while the lower ones, like fish and pigeons, tend to "probability learn". However, the experiments by Bitterman and his associates often use procedural features, such as forced trials to the alternatives, that complicate the calculation of the obtained frequencies of reinforcement, if not the psychological processes at work. In any event, pigeons do not invariably show probability learning; Herrnstein (1958) showed maximizing in pigeons given a choice between differing probabilities of reinforcement.

It is, in other words, not clear how much of the literature of probability learning actually violates equation 1 , since it is not clear how much of this literature can be taken as firm evidence for probability learning. Nevertheless, suppose for the sake of argument that there is some valid evidence for probability learning, which is to say, that the responses are in the same ratio as the probabilities of reinforcement. How does this differ from the findings with rate of reinforcement, according to which the responses are in the same ratio as the numbers of reinforcement? The two findings turn out to be closely related mathematically, as the following set of equations shows, starting with the equation for probability learning:

$$
\begin{aligned}
& \frac{\mathbf{P}_{\mathbf{L}}}{\mathbf{P}_{\mathbf{R}}}=\frac{\frac{\mathbf{R}_{\mathbf{L}}}{\mathbf{P}_{\mathbf{L}}}}{\frac{\mathbf{R}_{\mathbf{R}}}{\mathbf{P}_{\mathbf{R}}}} \\
& \mathbf{P}_{\mathrm{L}}{ }^{2} \mathbf{R}_{\mathbf{R}}=\mathbf{P}_{\mathrm{R}}{ }^{2} \mathbf{R}_{\mathrm{L}} \\
& P_{L} \sqrt{R_{B}}=P_{R} \sqrt{R_{L}} \\
& P_{L} \sqrt{R_{R}}+P_{L} \sqrt{R_{L}}=P_{R} \sqrt{R_{L}}+P_{L} \sqrt{R_{L}} \\
& \frac{\mathbf{P}_{\mathbf{L}}}{\mathbf{P}_{\mathrm{L}}+\mathbf{P}_{\mathrm{R}}}=\frac{\sqrt{\mathbf{R}_{\mathrm{L}}}}{\sqrt{\mathbf{R}_{\mathrm{L}}}+\sqrt{\mathbf{R}_{\mathrm{R}}}}
\end{aligned}
$$

Natapoff (in press) has shown that the matching of response frequencies to reinforcement frequencies (equation l) is just one, and in some sense the simplest, of a family of functions that may relate these two variables under the assumptions of symmetry of choice, which is to assume that the factors that affect choice operate invariantly across the alternatives.
Another, and closely related, function, according to Natapoff, would be equation 6 above, according to which the match is between response frequencies and the square root of reinforcement frequencies. This latter finding is, as the mathematical argument demonstrates, merely another version of probability learning. Although we do not know with certainty if, and when, probability learning actually occurs in simple choice procedures, it may eventually be useful to be able to relate the two kinds of matching to each other mathematically.

The only other significant departure from matching known to the present author is an experiment with pigeons (Herrnstein, 1958) in which reinforcement was dependent upon a required number of responses on the two keys. The sum of the two requirements was kept constant at 40 , but the two components varied: 2,$38 ; 5,35 ; 10,30$; and 20,20 . No further restrictions were imposed on the order or manner in which the requirements were reached. The pigeon could work in any sequence of alternation between the two keys, and could emit any number in excess of the requirement on one of the keys, but, having met one requirement, it was rewarded as soon as it met the other. Figure 5 shows the proportion of responses on the left key as a function of the proportion of the total requirement on that key. It also shows the proportion of reinforcements on that key. The procedure clearly produced a kind of matching, but not to the distribution of reinforcements, since the proportion of reinforcements was so much more variable than the proportion of responses. Instead, the pigeons responded so as to minimize the number of responses (and also probably the time) per reinforcement.

There are undoubtedly many other procedures that would give comparable departures from matching. For example, if reinforcement were made conditional upon a pair of rates of responding on the two keys (instead of upon a pair of numbers of responses- thereon), there probably would be some accommodation in responding and matching in the present sense would probably be violated. Such procedures need not be particularly complicated, the two examples so far notwithstanding. For example, consider a procedure that simply reinforced alternation of left and right responses, with reinforcement occurring only for right re- 


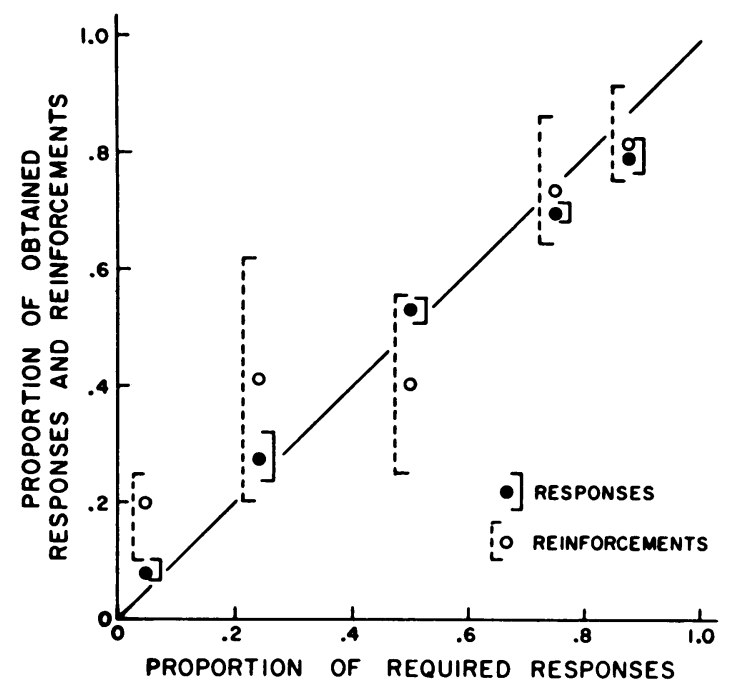

Fig. 5. The filled circles and solid brackets show the average and range of the relative frequency of responding to one alternative in a two-choice procedure. Reinforcement was for the occurrence of at least a minimum number of responses to each of the two alternatives. The abscissa shows what fraction of the total response requirement was allocated to the alternatives being plotted. The open circles and broken brackets are for the relative frequency of reinforcement against the same abscissa. The diagonal line shows matching between the abscissa and the ordinate. From Herrnstein (1958).

sponses. The distribution of responses would here approach 50-50, but the distribution of reinforcements would be $0-100$. This last example is instructive, for it suggests why matching neither occurs nor should be expected in such cases. Reinforcement for alternation is likely to give not two separate responses, but rather a single, albeit biphasic, responserespond-left-respond-right-that is reinforced every time it occurs. In the more complex case summarized in Fig. 5, there is a similar issue of response definition, as there is in any procedure in which reinforcement is conditional upon combinations of responses across the alternatives.

A procedure that reinforces multiphasic responses across the alternatives is thus not properly described by tallies of the individual response frequencies. Instead of so many left responses and so many right responses, there should be so many left-rights or the like. The point is worth noting for it is probably why matching depends on the COD in some (but not all) procedures. If the response alternatives are situated near each other, the subject's left-right or right-left sequences may be adventitiously reinforced. If so, the usual tally of left and right responses cuts across the response classes actually being maintained by the reinforcement and matching in the ordinary sense is no longer an appropriate expectation. By interposing a delay between a response to one alternative and reinforcement for the other, the COD discourages these adventitious response clusters.

In virtually all of the published experiments showing matching, the alternative responses were reinforced by schedules that differed only in the reinforcement parameter, whether frequency or amount. This may not, however, be necessary. For example, it remains to be shown whether matching would be found if the concurrent procedure used a variable-interval schedule pitted against a variable ratio. The procedure is peculiar not only because of its asymmetry, but also because it is intermediate as regards the degree to which the subject's behavior governs the distribution of reinforcement, and is therefore of some general interest. With two variable-interval schedules, the distribution of reinforcements is not likely to be affected by the distribution of responses, given the usual rates of responding. On the other hand, with two variable-ratio schedules, the distribution is virtually entirely under the subject's control. In the combination procedure, the animal is in control of reinforcement frequency for one alternative, but not for the other, again assuming that response rate is high enough. Finally, the experiment is peculiar because matching, while well established for interval schedules and their analogues, cannot occur for ratio schedules except trivially. For example, suppose ratios of 50 and 200 are scheduled for two alternatives. Equation 1, the matching function, can be satisfied only if the animal ceases responding to one of the alternatives, thereby obtaining all of its reinforcements thereon. The ratio schedules assure that the distribution of responses will follow the relation:

$$
\frac{P_{L}}{P_{L}+P_{R}}=\frac{50 R_{L}}{50 R_{L}+200 R_{R}}
$$

The coefficients on the reinforcement frequencies express the fact that a ratio schedule fixes a proportionality between numbers of responses and numbers of reinforcements. In the present example, equation 7 agrees with equa- 
tion 1 only when either $R_{L}$ or $R_{R}$ goes to zero. In general, agreement can be obtained only at these limiting values, plus the $50 \%$ value when the two ratios are equal to each other. In contrast, the present experiment, combining interval and ratio schedules, permits matching at all values, as follows:

$$
\frac{\mathbf{P}_{\mathbf{L}}}{\mathbf{P}_{\mathbf{L}}+\mathbf{P}_{\mathbf{R}}}=\frac{\mathbf{r R}_{\mathbf{L}}}{\mathbf{r R}_{\mathbf{L}}+\mathbf{x R _ { \mathrm { R } }}}
$$

Here, $r$ is the required proportionality for the alternative with the ratio schedule. For the other alternative, reinforced on a variableinterval schedule, there is no required proportionality. By responding faster or slower, the subject can here emit more or fewer responses per reinforcement $(x)$. In order to obtain agreement between equation 8 and equation 1 , the subject must adjust its rate of responding on the variable-interval schedule such that $\mathrm{r}=\mathbf{x}$.

An experiment combining the two schedules was performed with four pigeons. As in the earlier study, there was a change-over delay $(2 \mathrm{sec})$ between the two alternatives. Responding to one alternative was reinforced on variable-interval schedules that were changed from time to time (in the range of $15 \mathrm{sec}$ to $2 \mathrm{~min}$ ). Responding to the other was reinforced on variable-ratio schedules, also varied from time to time (in the range of 20 to 160). Since the distribution of reinforcements is here affected by the distribution of responses at all rates of responding, the values of the two schedules do not fix a particular distribution of reinforcement. The relative frequency of reinforcement, the nominal independent variable, was largely up to each subject. For most of the pairs of values, the findings trivially confirmed matching, by giving responding exclusively to one alternate or the other. This has also been the outcome when both alternatives were variable-ratio schedules (Herrnstein, 1958). However, for some pairs of values, the responding was distributed between the two responses with something between exclusive preference and total abstinence. Figure 6 shows the proportion of responses to one of the two alternatives (the variable-interval key) as a function of the proportion of reinforcements actually obtained from that key. The data for four pigeons have been averaged here, but the individual points were not systematically different. The finding is that even when the two alternatives reinforce according to different schedules, the distribution of responding still obeys the matching rule, or something close to it. The bunching of the points on the lower part of the function appears to be a reliable outcome of the procedure. It says that preferences for the variable-interval alternative (i.e., above 0.5 on the ordinate) are exclusive, while for the variable-ratio alternative (i.e., below 0.5 on the ordinate) they may be exclusive or non-exclusive.

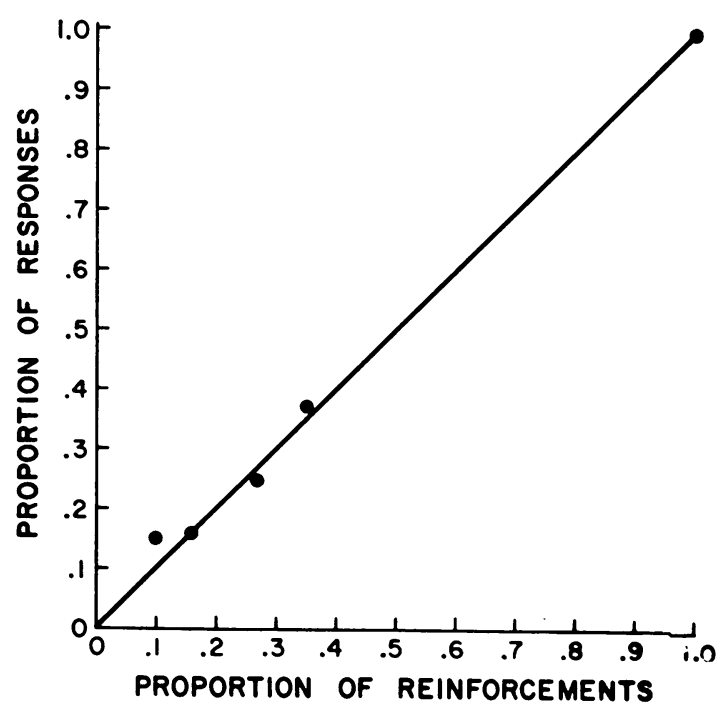

Fig. 6. The relative frequency of responding to one alternative in a two-choice procedure as a function of the relative frequency of reinforcement thereon. For one alternative, reinforcement was on a variableinterval schedule, while, for the other, it was on a variable-ratio schedule. The ordinate plots the variableinterval alternative. The diagonal shows matching between ordinate and abscissa.

The matching in Fig. 6 is surprising not only because of the peculiar procedure, but also in light of the well-known effects of the two kinds of schedules on responding in isolation. Variable-ratio responding is typically. a good deal faster than variable-interval responding, at equal frequencies of reinforcement (Herrnstein, 1964). In the present experiment, the momentary rates of responding were about twice as high for the ratio schedules as for the interval schedules. Nevertheless, each of the subjects maintained approximately the required invariance of responses per reinforcement across the ratio and interval schedules (see equation 8) even while the parameter values of both schedules were being changed. 


\section{CHOICE AS BEHAVIOR AND VICE VERSA}

Intuitively, response strength should covary in some reasonably orderly way with the parameters of reinforcement. The foregoing section presents the case for replacing intuition with the relative frequency of response. The argument is simply that since relative responding is so directly controlled by relative reinforcement, the former is the proper measure of the effects of the latter. The investigation of strength need not, however, stop here. Several theorists (Shimp, 1966; Neuringer, 1967a) have argued that matching is not fundamental, but is to be explained as the outcome of a more molecular interaction between behavior and its consequences, somehow based on "maximizing" in the probability-learning sense. The developments along this line will not be further examined here, for a persuasive case one way or the other is yet to be made. Suffice it to note that there is no logical assurance that this sort of reductionism will ever explain anything. The issue is empirical, and it is possible that behavior is more (or more simply) orderly at the level of the matching relation than at the level of interresponse times or sequences of choices, which is where the molecular theories operate. In contrast, logic demands that if the relative frequency of response is governed by the frequency of reinforcement, it must also govern somehow the absolute rate of responding. Since relative frequencies in the free-operant procedure are merely ratios of rates of responding, the one could hardly be affected without the other being affected as well.

The link between sheer output of behavior and what is usually called "choice" is peculiarly apparent with the free-operant method. In conventional choice experiments using discrete trials, the total number of responses is a trivial by-product of the procedure, depending simply on the number of trials per unit time. When the experimenter stipulates what the sheer output of behavior is going to be, he is unlikely to find it interesting. In the operant paradigm, however, where the output is as free to vary as anything else, if not more so, it becomes an interesting, and inescapable, dependent variable. Yet the matching relation holds for both continuous-responding and discrete-trial procedures. In the former, the question of sheer output cannot honestly be disregarded; in the latter, the question is precluded by the procedure. Since it seems unlikely that the two situations can be fundamentally different when they both produce matching, "choice" in the discrete-trial procedure should be explained the same way as the output of behavior for the free-operant procedure. It is hard to see choice as anything more than a way of interrelating one's observations of behavior, and not a psychological process or a special kind of behavior in its own right. For continuous-responding procedures, the correspondence between choice and behavior is clear, for the measure of choice is just the ratio of the simple outputs for the alternative responses.

What, then, can be said about these simple rates of responding? In the first concurrent procedure considered above (see Fig. 4), the two responses were freely occurring operants, reinforced on variable-interval schedules, and the matching relation was the ratio of their rates of occurrence. Figure 7 shows the absolute rates of responding of which the matching relation was the ratio. For absolute rates of responding there are twice as many degrees of freedom as for relative rates, since the two keys give values separately in the former in-

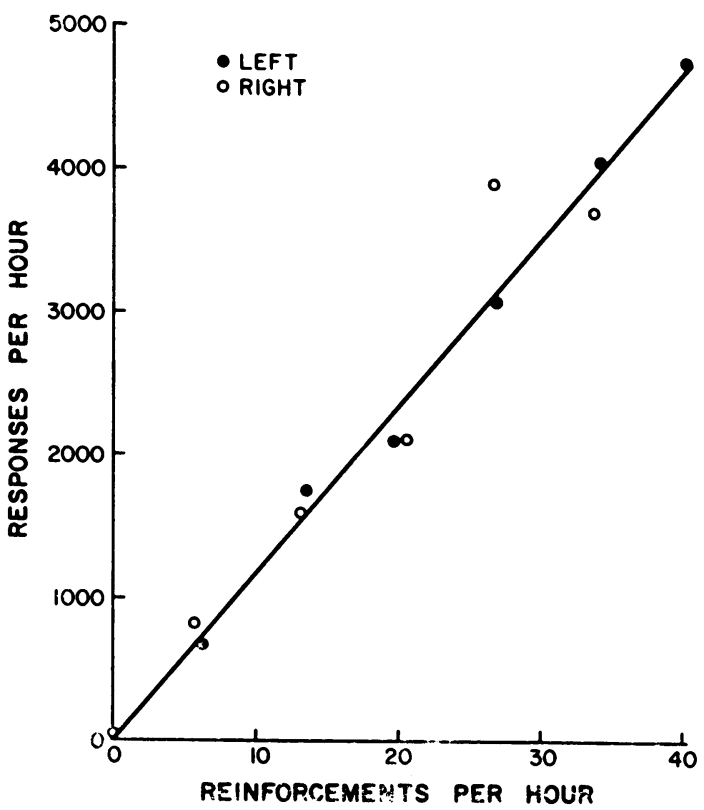

Fig. 7. The rate of responding for each of two alternatives in a two-choice procedure as a function of the rate of reinforcement for each. Variable-interval schedules were used for both. From Herrnstein (1961). 
stance, but complementarily in the latter. Even though the functions in Fig. 7 are, therefore, not as firmly demonstrated as in Fig. 4, the trend of the data is clear. (One point from the original experiment for one subject has been omitted since there are reasons to believe that it was aberrant.) The absolute rate of responding on each key is directly proportional to the absolute rate of reinforcement. This elegantly simple relation may be expressed in the equation:

$$
\mathbf{P}=\mathbf{k} \mathbf{R}
$$

Note that this relation is consistent with the basic matching relation, as it would have to be unless something were amiss. If each response alternative were obeying this rule, then any combination of responses with the same proportionality to reinforcement (i.e., the same k) should show matching, as simple algebra proves:

$$
\frac{\mathbf{P}_{\mathbf{L}}}{\mathbf{P}_{\mathrm{L}}+\mathbf{P}_{\mathbf{R}}}=\frac{k \mathbf{R}_{\mathrm{L}}}{k R_{\mathrm{L}}+\mathbf{k R _ { R }}}=\frac{\mathbf{R}_{\mathrm{L}}}{\mathbf{R}_{\mathrm{L}}+\mathbf{R}_{\mathbf{R}}}
$$

Equation 9 makes a plausible and impressively simple fundamental law of response strength. Its main trouble is that it has been tried before and failed, for, in another form, it merely restates Skinner's first published guess about response strength (1938, p. 130f), when he asserted the constancy of what he called the extinction ratio. From his early work, he surmised that the number of responses per reinforcement with interval schedules might be a constant, which is equation 9 solved for $\mathrm{k}$. Thus, if the animal's response is reinforced once every $5 \mathrm{~min}$ it should respond twice as fast as when the response is reinforced every $10 \mathrm{~min}$, and so on. Skinner's own data failed to support this simple principle, and later (1940) he revised the general principle of the "reflex reserve", so that the constancy of the extinction ratio was no longer of any theoretical importance in his system. The mass of data collected since shows no such relation as equation 9 in single-response procedures, as Catania and Reynolds (1968) demonstrated most exhaustively.

In the experiment summarized in Fig. 7, the over-all frequency of reinforcement was held constant at 40 per hour while the proportion allocated to one alternative or the other was varied. As long as the total number of reinforcements $\left(R_{L}+R_{R}\right)$ is constant, there is no way to distinguish in a single experiment between the prediction of equation 9 and the following:

$$
P_{L}=\frac{k R_{L}}{R_{L}+R_{R}}
$$

When the sum $R_{L}+R_{R}$ is itself a constant, equation 11 is equivalent to equation 9 , the only difference being the value of $k$, which is an arbitrary constant in either case. As regards Fig. 7, then, equation 11 is as good as equation 9. The two equations make divergent predictions only when $R_{L}+R_{R}$ is not a constant, which is actually more typical in concurrent procedures. The difference is that equation 11 predicts a "contrast" effect, which is to say a reciprocal relation between the reinforcements for one alternative and the responses to the other, whereas equation 9 predicts independence of responding to one alternative and reinforcements for the other. The data unequivocally support equation 11, for contrast effects are reliably found in concurrent procedures (Catania, 1966).

Although equation 11 conforms at least qualitatively to the literature on concurrent procedures, it, like equation 9 , seems to run into trouble with single-response situations, for when there is only one response alternative being reinforced the equation degenerates into the following constancy:

$$
\mathbf{P}=\mathbf{k}
$$

Responding in single-response situations is notoriously insensitive to variations in the parameters of reinforcement, but it is not totally insensitive. Equation 12, however, is the result of a gratuitous assumption, albeit one that is easily overlooked. It assumes that because the experimenter has provided just one response alternative, there is, in fact, just one. A more defensible assumption is that at every moment of possible action, a set of alternatives confronts the animal, so that each action may be said to be the outcome of a choice. Even in a simple environment like a single-response operant-conditioning chamber, the occurrence of the response is interwoven with other, albeit unknown, responses, the relative frequencies of which must conform to the same general laws that are at work whenever there are multiple alternatives. In fact, it seems safe to assume that all environments continually demand choices in this sense, even though in 
many cases the problem of identifying and measuring the alternatives may be insoluble. That problem is, however, the experimenter's, not the subject's. No matter how impoverished the environment, the subject will always have distractions available, other things to engage its activity and attention, even if these are no more than its own body, with its itches, irritations, and other calls for service.

The notion that started this section, that choice is not a psychological mechanism so much as a certain measure extracted from observations of behavior, has a complement. For while choice is nothing but behavior set into the context of other behavior, there is no way to avoid some such a context for any response. An absolute rate of responding is occurring in such a context, whether or not the experimenter happens to know what the other alternatives and their reinforcers are. With this in mind, equation 11 may be rewritten for a nominal single-response situation, but recognizing the possibility of other sources of reinforcement, as follows,

$$
\mathbf{P}=\frac{\mathbf{k R}}{\mathbf{R}+\mathbf{R}_{\mathbf{0}}}
$$

in which $R_{0}$ is the unknown, aggregate reinforcement for other alternatives. ${ }^{2}$ In practical terms, $R_{0}$ is a second free parameter to be extracted from the data, but it is one that has a definite empirical interpretation. The question is whether equation 13 fits the data.

Figure 8 is a test of equation 13 with data obtained by Catania and Reynolds (1968). The six pigeons in the experiment were submitted to variable-interval schedules with rates of reinforcement ranging from about 10 to about 300 per hour. The effects on performance were varied, as the points in Fig. 8 show. However, with few exceptions, the points fall on or near the smooth curves, which are plots of equation 13. The parameter values for the individual subjects are shown in each panel, $k$ first and $R_{o}$ second, with $k$ in responses per

${ }^{2}$ This same equation for absolute rate of responding in single-response situations can be found in Norman (1966), except that Norman offers the equation as an approximation, rather than as an exact relationship between the variables. Norman's analysis does not appear to be applicable to multiple-response situations and his interpretation of the parameters is entirely different from the present one. The two accounts are, therefore, readily distinguishable, notwithstanding the convergence at this point.

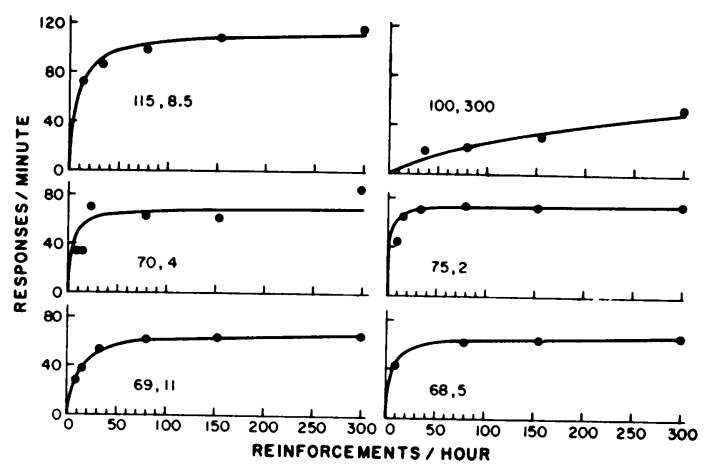

Fig. 8. The rate of responding as a function of the rate of reinforcement for each of six subjects on variable-interval schedules. The first number in each panel is $k$ in responses per minute and the second is $R_{0}$ in reinforcements per hour for the various smooth curves. From Catania and Reynolds (1968).

minute and $\mathbf{R}_{\mathrm{o}}$ in reinforcements per hour. The fit of points to function for each animal shows that the mathematcial form of equation 13 is suitable for the single-response situation, notwithstanding its origin within the context of choice experiments. The variety of parameter values further shows that inter-subject variability is also amenable to description in these formal terms. There appears to be no other comparably simple mathematical expression for the relation between absolute input and output for simple, repetitive responding, let alone one that also predicts the distribution of choices over multiple alternatives.

Figure 9 is another test for equation 13, showing the results of an unusual procedure for varying reinforcement frequency. The parameter values are again for $k$ in responses per minute and $R_{0}$ in reinforcements per hour, in that order. Chung (1966) reinforced his pigeons' responses after a given duration had elapsed since the first response after the last reinforcement-in the terminology of reinforcement schedules, a tandem fixed ratio 1 , fixed interval of various durations. From time to time, the duration was changed, giving a new determination of rate of responding. The actual rate of reinforcement was substantially controlled by the subjects, for the sooner after reinforcement the animals responded, the sooner the required time interval would begin. Chung's experiment is worth noting here not only because the procedure is unusual, but because it achieved reinforcement rates of about 2000 per hour, seven times Catania and Reynolds' (1968) maximum. Nevertheless, 


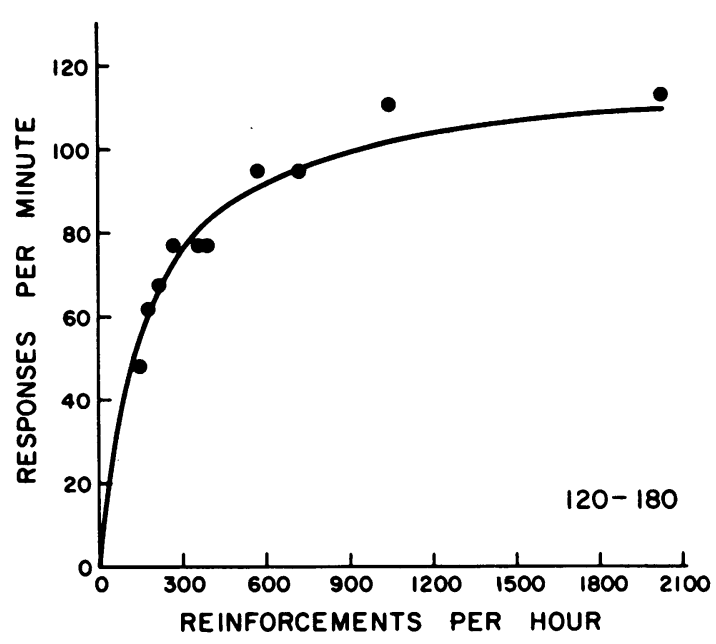

Fig. 9. The rate of responding as a function of the rate of reinforcement for subjects on a tandem fixedratio, fixed-interval schedule. The first number is $k$ in responses per minute and the second is $R_{0}$ in reinforcements per hour for the smooth curve. From Chung (1966).

Chung's results are well described by equation 13, although the parameter value for $\mathbf{R}_{0}$ (180) is substantially higher here than for five of Catania's and Reynolds' six subjects.

Equation 13 is readily expanded into a general principle of response output, whether the response is presumably in isolation or not. The matching relation can be derived by taking into account the reinforcement from both alternatives:

$$
\begin{aligned}
& \frac{\mathbf{P}_{\mathbf{L}}}{\mathbf{P}_{\mathbf{L}}+\mathbf{P}_{\mathbf{R}}}=\frac{\frac{\mathbf{k} \mathbf{R}_{\mathbf{L}}}{\mathbf{R}_{\mathbf{L}}+\mathbf{R}_{\mathbf{R}}+\mathbf{R}_{\mathbf{o}}}}{\frac{\mathbf{k} \mathbf{R}_{\mathbf{L}}}{\mathbf{R}_{\mathbf{L}}+\mathbf{R}_{\mathbf{R}}+\mathbf{R}_{\mathbf{o}}}+\frac{\mathbf{R}_{\mathbf{R}}+\mathbf{R}_{\mathbf{R}}+\mathbf{R}_{\mathbf{0}}}{\mathbf{R}_{\mathrm{L}}}} \\
& =\frac{\mathbf{R}_{\mathbf{L}}}{\mathbf{R}_{\mathbf{L}}+\mathbf{R}_{\mathbf{R}}}
\end{aligned}
$$

This assumes that $k$ and $R_{0}$ are the same for the responses under observation, a reasonable supposition as long as the responses are equivalent in form and effort. If choice were, however, asymmetric-for example, if one response was to depress a lever while the other was to pull a chain-the equivalence of neither $k$ nor perhaps $\mathbf{R}_{0}$ could be assumed. Matching would not be predicted, but the present formulation would apply in principle nevertheless.

In recent experiments on choice, the symmetry assumption has been supportable, which is to say, the matching relation has been found. The next question is whether the absolute rates of responding also conform to the present formalization. Equation 14 contains the expressions for the absolute rates of responding, restated here for convenience (for the left key):

$$
\mathbf{P}_{\mathrm{L}}=\frac{\mathbf{k} \mathbf{R}_{\mathrm{L}}}{\mathbf{R}_{\mathrm{L}}+\mathbf{R}_{\mathrm{R}}+\mathbf{R}_{\mathbf{0}}}
$$

For the first study of matching (Herrnstein, 1961), the agreement between fact and theory is clear, since that is where the discussion began (see Fig. 7). When the total reinforcement is held constant, as was the case in that experiment, the absolute rate of responding should be directly proportional to the absolute rate of reinforcement. When the over-all rates of reinforcement are not constant, there should be "contrast" effects, with the rate of responding on each alternative varying directly with the associated rate of reinforcement, but inversely with the rates of reinforcement elsewhere.

Catania's work (1963b) provides a quantitative evaluation of equation 15. Catania used pigeons on the Findley procedure. The two alternatives were the usual variable-interval schedules, each one signalled by a particular color on the response key. The variable intervals ran concurrently, but, in accordance with the Findley procedure, there was only one color (and, therefore, but one variable interval) making direct contact with the subject at any one time. A peck on the second key changed the color and brought the second variable interval into contact with the subject. The difference between this sort of concurrent schedule and the more familiar type is in the "switching" response: for the Findley procedure it is the pecking of a key, for the other, it is actually moving back and forth between the keys. Figure 10 shows that the difference does not affect the matching relation. Both the proportion of responses and the proportion of time equal the proportion of reinforcement for each alternative.

Equation 15, however, calls for absolute rates of responding, not proportions, and these are plotted in Fig. 11. There were two series of reinforcement rates in the experiment. In one, the sum of the reinforcement rates for the two alternatives was always 40 per hour, just as in Fig. 7 (Herrnstein, 1961). In the other, the sum varied, but was held at 20 per hour for one of the two schedules. Equation 15 predicts a direct proportionality 


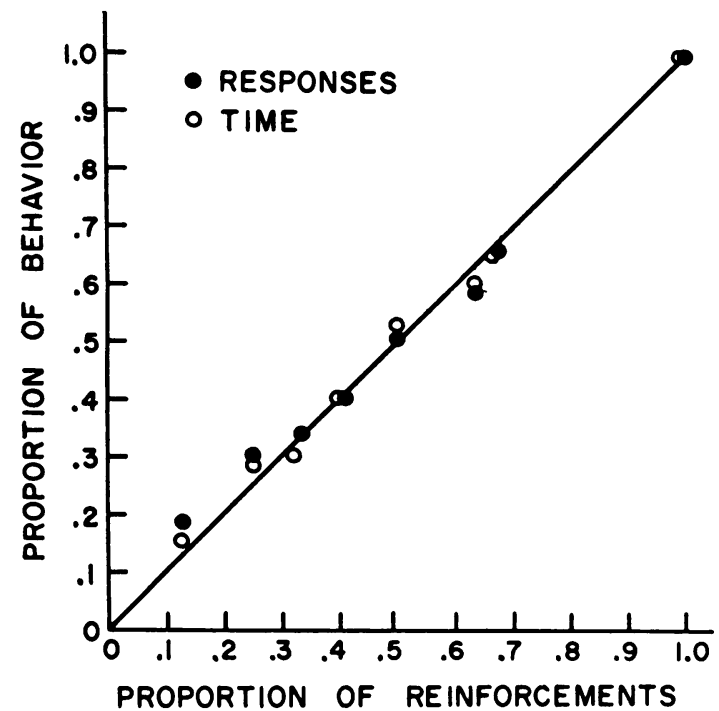

Fig. 10. The relative number of responses emitted (filled circles) and the relative amount of time spent (open circles) on one alternative in a two-choice procedure as a function of the relative frequency of reinforcement thereon. The diagonal shows matching between ordinate and abscissa. From Catania (1963b).

for the first condition and a curvilinear relation for the second. The left portion of Fig. 11 shows responding as a function of reinforce-

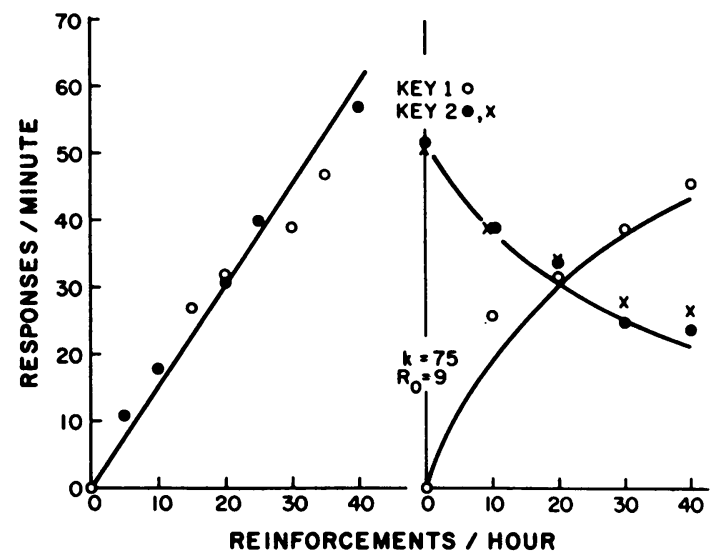

Fig. 11. The rate of responding as a function of the rate of reinforcement for each alternative in a twochoice procedure. For the left panel, the overall frequency of reinforcement was held constant at 40 reinforcements per hour, while varying complementarily for the two alternatives. Each point is here plotted above the reinforcement rate at which it was obtained. For the right panel, the frequency of reinforcement for Key 2 was held constant at 20 reinforcements per hour, while varying between 0 and 40 for Key 1. The points here are plotted above the reinforcement rate on Key 1 at the time it was obtained. The values of $k$ and $\mathbf{R}_{\mathrm{o}}$ were used for the smooth curves in both panels. From Catania (1963b). ment when the total reinforcement rate was held constant at 40 per hour. For each alternative, the points approximate a straight line passing through the origin, as theory predicts. The selected line has the parameters $k=75$ (responses per minute) and $\mathbf{R}_{\mathrm{o}}=9$ (reinforcements per hour). Note that these values are easily within the range obtained in Fig. 8 for a single-response situation involving variableinterval schedules. The right-hand panel shows responding as a function of reinforcement rate on Key $1^{3}$ for the second series, when the reinforcement for one key (Key 2) was held constant at 20 per hour while Key 1 reinforcements ranged from 0 to 40 per hour. The contrast effect is the decreasing rate on Key 2 as reinforcement for Key 1 increases. The smooth curves again plot equation 15, with the parameters again at $k=75$ and $R_{0}=9$. The crosses on the right panel show the results of a second procedure with the same three pigeons. Responding to Key 2 was still reinforced 20 times per hour, while that to Key 1 was again varied from 0 to 40 per hour. The change was that the pigeons switched to Key 1 only when a light signalled that reinforcement was due there, so that the number of responses to Key 1 was virtually equal to the number of reinforcements. Otherwise, the procedure was unchanged. As the decreasing rate of Key 2 responding shows, and as equation 15 implies, contrast depends upon the reinforcement for the other alternative, not on the responding there. The crosses may actually deviate slightly from the filled circles, for reasons that will be considered in the next section, but aside from this, Catania's data provide substantial confirmation for Equation 15 and the system from which it emerges.

Catania accounted for his results with a power function between absolute responding and the relative number of reinforcements, as follows:

$$
P_{1}=\frac{k R_{1}}{\left(R_{1}+R_{2}\right)^{5 / 8}}
$$

Like equation 15, this one, too, predicts matching when $\mathbf{k}$ and the denominators can be cancelled out, which is to say, when choice

${ }^{3}$ With the Findley procedure, the designations, "Key 1 " and "Key 2", do not identify two separate response keys. Rather, they represent the two possible states of the key to which responses were reinforced. 
is between otherwise equivalent alternatives. Given the variability in the data, it would be hard to choose between equation 16 and the present formulation as contained in equation 15. The most easily testable difference between Catania's hypothesis and the present one is in regard to responding in single-response situations, for which equation 16 implies a power function with an exponent of $1 / 6$, while equation 15 implies equation 13. The data, the best of which we owe to Catania and Reynolds (1968), distinctly favor the latter alternative, since no power function handles the data from individual pigeons in single-response procedures while equation 13 makes a plausible fit, as shown in Fig. 8 and 9.

Equation 13 is for single-response procedures; equation 15, for two-response procedures. The general case, for a situation containing $\mathbf{n}$ alternative sources of reinforcement, is:

$$
P_{1}=\frac{k R_{1}}{\sum_{1=0}^{n} R_{1}}
$$

This mathematical form is not used throughout because it hides $R_{0}$, the parameter in the denominator. It does, however, reveal the most general implications of the theory. Matching will take place over any set of alternative responses for which $k$ and $\Sigma R$ are fixed, the typical choice procedures, for example. Inversely, when matching is not obtained, the alternatives either have different asymptotic rates (k) or are operating within different contexts of reinforcement $(\Sigma R)$, or both. It is not hard to conceive of situations that exhibit either or both of these asymmetries, even though they are somewhat unfamiliar in experiments on choice. The next section summarizes research in a field that shows the latter asymmetry, the absence of a fixed context of reinforcement.

The idea of a "context" of reinforcement is fundamental to the present analysis, for that is what the denominator is best called. Equation 17 says that the absolute rate of responding is a function of its reinforcement, but only in the context of the total reinforcements occurring in the given situation. When the total is not much more than the reinforcement for the response being studied, then responding will be relatively insensitive, for the ratio $R_{1} / \Sigma R_{1}$ will tend toward 1.0. This ex- plains, at least partly, why for over three decades, interesting functional relations between reinforcement and responding have been so scarce. Experimeters have naturally tried to make the behavior they were observing important to the subject, and they have probably succeeded. If the response was to be reinforced with food or water, then the state of deprivation, the size of the reinforcer, and the experimental isolation of the subject have probably guaranteed that $\Sigma R$ will be only negligibly larger than $R_{1}$. To do otherwise, which is to make $\Sigma R$ substantially larger than $R_{1}$, is to risk unexplained variability, for then $P_{1}$ will be at the mercy of fluctuations in the other (usually unknown) reinforcers in the situation. Investigators have made a tacit decision in favor of stability, but at the cost of sensitivity to the independent variable.

Equation 17 explains the unique advantage of concurrent procedures. Because $\Sigma R$ and $k$ are eliminated when comparable responses are being measured as relative frequencies, the stability-sensitivity dilemma is avoided. The peculiar discrepancy between single and multiple response situations has been noted, with the one stubbornly insensitive and the other gratifyingly orderly and interesting (Catania, 1963a; Chung and Herrnstein, 1967). For magnitude, frequency, and delay of reinforcement, it has been shown that matching in multipleresponse procedures and virtual insensitivity in single-response procedures are the rule. The discrepancy is a corollary of equation 17, which implies that responses at comparable levels of strength, as in symmetric choice experiments, will not only be sensitive to shifts in reinforcement between them, but equally affected by uncontrolled changes in extraneous reinforcers and therefore relatively orderly with respect to each other.

\section{INTERACTION AT A DISTANGE}

Equation 17 says that the frequency of responding is proportional to its relative reinforcement. The interval over which these responses and reinforcements are counted has so far been taken for granted. In simple choice, the interval is the experimental session itself, which is usually homogeneous with respect to rates of reinforcement and responding. The literature of operant conditioning contains, however, many experiments in which neither 
behavior nor its consequences are evenly distributed over sessions, and many of these experiments show interactions between successive (as well as simultaneous) conditions of reinforcement. An early and clear instance was Reynolds' (1961a) experiment, in which the rate of responding by pigeons on a variableinterval schedule increased when the schedule alternated with a period of non-reinforcement. The procedure started with 3-min alternation between red and green light on the response key, but responding was reinforced on the variable-interval schedule throughout. As expected, the rate of responding was approximately steady. When reinforcement was discontinued during, let us say, the red periods, responding during the green periods rose by about $50 \%$, even though the rate of reinforcement was unchanged therein. In another part of the experiment, a variable-ratio schedule was used instead of a variable-interval schedule, with comparable results.

This "contrast" effect, as Reynolds termed it, has been widely confirmed, under many circumstances. For present purposes, the question is whether or not such changes in rate of responding belong with the present account of response strength. To anticipate the answer, the conclusion will be affirmative: the contrast effect is largely, if not entirely, derivable from an adaptation of equation 17.

If Reynolds had used a concurrent procedure, instead of a multiple schedule, a contrast effect would have been in order. With a variable interval of $3 \mathrm{~min}$ (VI 3-min) for responding on each key, the rate of responding is governed by the equation (measuring in reinforcements per hour),

$$
P=\frac{k 20}{40+R_{0}}
$$

With extinction on the other key, the rate on this key should rise, since it would now be governed by,

$$
P=\frac{k 20}{20+R_{0}}
$$

If $R_{0}$, the unscheduled, extraneous reinforcement, is assumed to be vanishingly small, the contrast effect is a doubling, i.e., $100 \%$. The larger is $R_{0}$, the smaller is the contrast effect. In Reynolds' experiment, the contrast effect was only about $50 \%$, which implies $R_{o}=20$ reinforcements per hour, assuming, for the moment, that equations 18 and 19 are appropriate. Although $\mathbf{R}_{\mathbf{o}}$ may have been this large in his experiments, the more typical range for pigeons in the standard chamber is 1 to 10 , with only infrequent instances falling outside. This, and other considerations, suggest that the multiple schedule, in which the alternatives succeed each other, differs in some way from simultaneous choice as regards the analysis of response strength.

For simultaneous choice, each source of reinforcement is assumed to exert a full effect on every response alternative. However plausible this assumption is for concurrent procedures, it is less so for multiple schedules, in which the various sources of reinforcement are not simultaneously operative. As the components of a multiple schedule become more separate, the interaction across components is likely to diminish; in the limiting case, diminish to no interaction at all. Thus, if the components alternate slowly and are denoted by distinctive stimuli, the interaction might be smaller than if the alternation is rapid and the stimuli marginal.

There are many ways to translate this continuum of interactions into a formal expression, but one of the simplest is to assume that the reinforcement in one component affects the responding in the other by some constant fraction of its full effect. For the two-component multiple schedule, then, the rate of responding in one component would be given by: 4

$$
P_{1}=\frac{k R_{1}}{R_{1}+m R_{2}+R_{0}}
$$

This is identical to the equation for one of the alternatives in a two-choice procedure

"Note that the time base for calculating the rate of responding in multiple schedules is the duration of the component during which the responding may occur. For concurrent schedules, the time base is the entire experimental session (excluding feeding cycles, timeouts, etc.). In general, the time base for calculating a rate of responding should be the time during which responding may occur, which differs as indicated in multiple and concurrent procedures. Also, note that the quantity $R_{0}$ in equation 20 represents a composite of the extraneous reinforcement during one component (component \#1 in the example in the text) plus the extraneous reinforcement in the other component, with the latter suitably decremented by the multiplicative factor $m$. In a fuller account, $R_{0}$ should be written out as $R_{01}+{ }_{m} R_{02}$. Such detail is clearly premature at this point. 
(equation 15), except for the additional parameter, $m$, which should vary between 0 and 1.0, depending upon the degree of interaction across components. Equation 15 may, in fact, be considered a special case of equation 20 , where $\mathrm{m}=1$.

The remainder of this section is a consideration of multiple schedules along the lines implied by equation 20 . It should be noted at the outset that this equation is just one way to extend the present formulation to situations in which interactions are sub-maximal. As the following discussion shows, the data are not quite adequate either to confirm equation 20 or to demand an alternative. For present purposes, however, it suffices to demonstrate that many of the effects of multiple and concurrent procedures follow from a single conception of response strength.

Equation 20 assumes not only a simple mechanism to govern the degree of interaction, but it tacitly describes the most symmetrical sort of multiple schedule, as shown by the absence of subscripts on the parameters, $k, m$, and $R_{o}$. This is tantamount to assuming that the response alternatives have equal asymptotic rates ( $k)$, that the interaction one way is the same as the interaction the other $(\mathrm{m})$, and that the unscheduled reinforcement is the same during the two components $\left(R_{0}\right)$. This is for a multiple schedule, in other words, with the same response-form in both components (k), with the same rule for the alternation from one component to the other as for the alternation back (m), and with the extra-experimental factors held constant $\left(\mathbf{R}_{\mathbf{o}}\right)$. It is possible to violate any of these conditions, but that should only complicate the analysis, not change it. Reynolds' experiment appears to satisfy these restrictions, and his results would follow from equation 20 if $\mathrm{m}$ was between 0.55 and 0.75 , assuming $R_{0}$ between 1 and 10 reinforcements per hour.

Equation 20 says that the contrast effect depends upon the reinforcement in the other component. Reynolds addressed himself to this issue at an early stage $(1961 b)$. He showed contrast when a "timeout" period was substituted for extinction. During a timeout, pigeons do not peck the key, and, in Reynolds' experiment, they received no reinforcement. Timeout and extinction should have the same effects on responding in the other component if they both reduce reinforcement in the inter- acting component to zero, and if equation 20 is correct. Reynolds then went on to show that as long as reinforcement in the interacting component is sustained, with or without responding, contrast is prevented. In one procedure, reinforcement was for $50 \mathrm{sec}$ of non-responding, a technique that suppresses responding, but does not eliminate reinforcement. Contrast was not observed. As Reynolds concluded, and as equation 20 implies, "The frequency of reinforcement in the presence of a given stimulus, relative to the frequency during all of the stimuli that successively control an organism's behavior, in part determines the rate of responding that the given stimulus controls." (p. 70, his italics)

The importance of reinforcement rate is clearly shown in Bloomfield's study of two multiple schedules (1967a). One multiple schedule consisted of 2-min alternations of VI 1-min with a DRL, the latter a procedure in which the response is reinforced only after specifed periods (varied from 5 to $15 \mathrm{sec}$ ) of non-responding. In the other schedule, VI 1min alternated with a fixed-ratio schedule (varied from 10 to 500). The two halves of the experiment shared a standard variable-interval schedule alternating with a schedule that yielded various different rates of reinforcement. Moreover, for both fixed-ratio and DRL schedules, the rate of reinforcement is a continuous joint function of the emitted rate of responding and the experimental parameter. However, the difference is that the functions are opposite, for on a fixed-ratio schedule, the rate of reinforcement is proportional to the rate of responding, while on the DRL, the rate of reinforcement is inversely related to the rate of responding given the levels of responding obtained by Bloomfield. Nevertheless, both of Bloomfield's multiple schedules support the implication of equation 20 , that reinforcement rate determines contrast. The rate of responding during the variable-interval component was an inverse function of the rate of reinforcement in the other component, without regard to whether the other schedule was a fixed ratio or a DRL. Neither the direction nor the magnitude of the contrast effect was apparently affected by the rate of responding or the schedule per se in the interacting component.

The question now is whether the dependence of contrast on reinforcement is in quan- 
titative, as well as qualitative, agreement with equation 20. Reynolds (1961c), using multiple VI FR schedules, concluded not only that the relative rate of responding was a function of the relative rate of reinforcement, but that the function was linear, with a positive intercept and slope less than 1.0. This was to be distinguished from concurrent procedures, where the matching relation holds (slope $=1.0$; intercept $=0$ ).

Reynolds' linear relation is at variance with equation 20. The predicted relation between the relative frequency of responding and the relative frequency of reinforcement is considerably more complex than linear, as follows: ${ }^{5}$

$$
\begin{aligned}
\frac{\mathbf{P}_{1}}{\mathbf{P}_{1}+\mathbf{P}_{2}} & =\frac{\frac{k R_{1}}{\mathbf{R}_{1}+m R_{2}+\mathbf{R}_{0}}}{\frac{k R_{1}}{\mathbf{R}_{2}+m R_{2}+R_{0}}+\frac{k R_{2}}{R_{2}+m R_{1}+R_{0}}} \\
& =\frac{1}{1+\frac{R_{2}\left(R_{1}+m R_{2}+R_{0}\right)}{R_{1}\left(R_{2}+m R_{1}+R_{0}\right)}}
\end{aligned}
$$

As a function of the relative frequency of reinforcement, $R_{1} /\left(R_{1}+R_{2}\right)$, this expression plots as a family of reverse-S-shaped functions, the curvature of which is dependent upon the relative magnitudes of $R_{1,2}, R_{0}$, and $m$. Complex as this is, it would be even worse without the assumptions of symmetry that qualify equation 20. The more general formulation will not, however, be explicated here, since there appear to be virtually no data to test it. Figure 12 shows a representative sampling from the family expressed in equation 21 . The ordinate is the relative rate of responding in one component of a multiple schedule and the abscissa is the relative rate of reinforcement therein. When the interaction between components is absent, which is to say, when there is no contrast effect, then the parameter $m=0$. When interaction is maximal, as in concurrent procedures, $m=1.0$. The shape of the function depends jointly on the degree of interaction (m) and on $R_{0}$, which is the reinforcement from sources other than the multiple schedule itself. If both this quantity and the interaction term, $\mathrm{m}$, go to zero, then the relative rate of responding during a component will be insensitive to changes in the rela-

\footnotetext{
${ }^{5}$ This assumes that the quantities $P_{1}$ and $R_{1}$ are either absolute numbers taken over equal periods of time or are themselves rates.
}

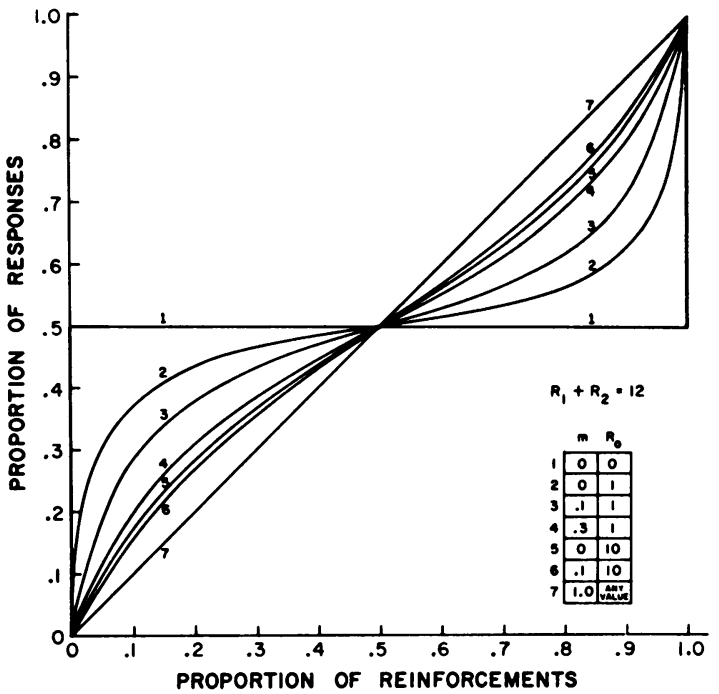

Fig. 12. Hypothetical curves relating the relative frequency of responding and the relative frequency of reinforcement in a two-component multiple schedule. The rate of reinforcement summed across the two components is assumed to be held constant at 12 reinforcements per unit time, while the other parameters vary as indicated on the figure. See text for discussion of equation 21 .

tive rate of reinforcement. For any given value of $\mathrm{m}$, the function is made steeper by larger values of $R_{0}$. As long as $m$ is less than 1.0 , the function approaches the matching line (7) asymptotically as $R_{o}$ increases. On the other hand, with $m=1.0$, the matching line is obtained, notwithstanding the value of $R_{0}$. The effect of $R_{0}$ at $m<1.0$ depends upon the relative magnitudes of $R_{0}$ and the over-all scheduled rates of reinforcement, which is 12 reinforcements per unit time for Fig. 12. When $R_{o}$ is relatively small, it exerts a smaller effect than when it is relatively large.

Points on the functions in Fig. 12 may easily be taken for linear, particularly if they fall in the middle of the range, as Reynolds' (1961c) did. The most complete set of data to test equation 21 is Reynolds' experiment $(1963 b)$ on multiple VI VI. Three pigeons were each run through three sequences of values. In the first, one of the schedules was held constant at VI 3-min while the other varied; in the second, both varied; in the last, one was again held constant, but at VI 1.58min. Figure 13 shows for each pigeon how well equation 21 handles the data for all three series. The smooth curves plot equation 21, with different parameters for the three pi- 


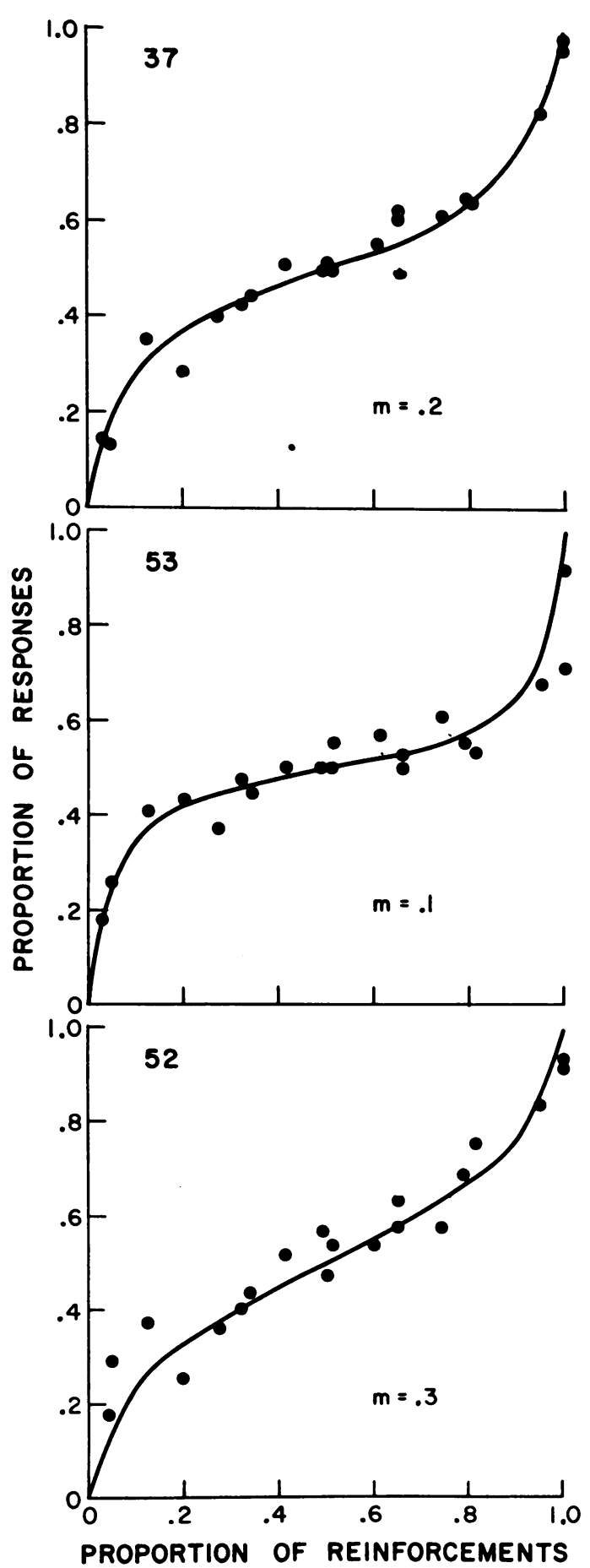

Fig. 13. The relative frequency of responding as a function of the relative frequency of reinforcement in one component of a multiple variable-interval, variable-interval schedule, for each of three subjects. The smooth curves plot the theoretical functions, with $\mathbf{R}_{\mathrm{o}}=\mathbf{0}$ and $\mathrm{m}$ set at the values indicated. From Reynolds (1963b). geons. For 37, $\mathrm{m}=0.2$; for $52, \mathrm{~m}=0.3$; and for $53, \mathrm{~m}=0.1 . \mathrm{R}_{\mathrm{o}}$ was arbitrarily set at 0 for all pigeons for convenience in calculation and presentation. If non-zero values of $R_{0}$ had been used, then no single predicted curve could have been drawn for each pigeon, since the over-all frequency of reinforcement was not held constant, and the effect of $R_{o}$ depends upon the over-all frequency of reinforcement. In any case, the typical value of $R_{0}$ can exert only negligible effects on the relative rate of responding, at these rates of reinforcement. The fit of the points to the data justifies both the omission of $R_{o}$ and the general formulation underlying equation 21 .

Although the fit in Fig. 13 supports the present account, reverse-S curves are not necessarily evidence for contrast. Figure 12 shows that curves of this general shape may be obtained even when $m=0$, which is to say, when the reinforcement in each. component affects the responding in that component only. To show contrast effects directly, absolute, not relative, rates of responding are in order. Although Reynolds' absolute rate data contain evidence for contrast, they were too variable, both within and between subjects, to permit a useful test of the present formulation. The most comprehensive data for this purpose appear to be in an unpublished experiment by J. A. Nevin.

Nevin used pigeons on a three-component multiple schedule, of which two were conventional VI 1-min and VI 3-min, signalled by the color of the illumination on the response key. Each lasted for $1 \mathrm{~min}$, after which the chamber was darkened for $30 \mathrm{sec}$. The independent variable was the rate of reinforcement during the timeout, which was set at five nominal values, from zero to one every $10 \mathrm{sec}$. The absolute rate of responding during a component should follow from equation 20, modified to take into account the presence of three, instead of two, components. For the component with VI 1-min (60 reinforcements per hour), the equation is, to a first approximation,

$$
P_{1}=\frac{k 60}{60+m\left(\frac{20+x}{2}\right)+R_{0}} ;
$$

for the VI 3-min (20 reinforcements per hour) component, it is

$$
P_{2}=\frac{k 20}{20+m\left(\frac{60+x}{2}\right)+R_{0}} .
$$


The quantity, $\mathbf{x}$, is the independent variable, which is to say, the reinforcement rate during timeout. The interaction term, $\mathrm{m}()$, is the average rate of reinforcement during the two components that might cause contrast. This formulation disregards at least two factors that are likely to have some effect. First of all, it tacitly assumes equivalent interactions between responding in a given component and reinforcement in either of the two other components. A more realistic assumption would be that the component closer in time exerts a greater effect, as, in fact, some work has indicated (Boneau and Axelrod, 1962; Pliskoff, 1963). Secondly, it tacitly assumes that the durations of the components $(1 \mathrm{~min}$ as opposed to $30 \mathrm{sec}$ for the timeout) are immaterial as regards contrast, whereas a component's effect probably increases with its duration. Placing values on these two complicating factors would be totally arbitrary at present, as well as somewhat unnecessary, since they may at least be presumed to operate in opposite directions, the nearer component being the briefer.

Figure 14 shows the absolute rate of responding during the two variable-interval components, averaged over the four subjects. The two curves are plots of equations 22 and 23. Narrow bands, rather than lines, are drawn for the predicted values because of variation in the obtained rates of reinforcement on the

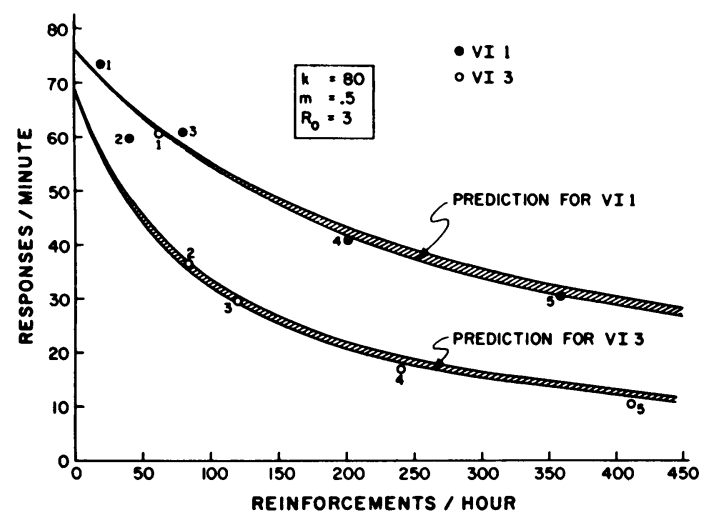

Fig. 14. The rate of responding in one component as a function of the rate of reinforcement summed across the other two components in a three-component multiple schedule. The filled circles and the upper curve are for the component held constant at a variable interval of $3 \mathrm{~min}$. Both curves drawn with lower curve are for the component held constant at a variable-interval of $3 \mathrm{~min}$. Both curves drawn with the parameters as indicated in the box. From Nevin (unpublished). nominal VI 1-min and VI 3-min. The abscissa is the rate of reinforcement summed across two of the three components, omitting the component from which responding is plotted (i.e., two times the term in parenthesis in equations 22 and 23). The numbers alongside the points identify the two values from each of the five multiple schedules studied. Generally, the point on the lower curve is shifted 40 reinforcements per hour to the right of the upper point, which is the difference between the VI 1-min and the VI 3-min. These decreasing functions are pure contrast, since the rates of reinforcement were close to constant in each of the variable-interval components (except for the last point on the lower curve). Equations 22 and 23, with parameters set at $R_{o}=3$ reinforcements per hour, $m=0.50$, and $k=80$ responses per minute, appear to be satisfactory except for the first point for VI 3-min.

Contrast is generally (e.g., Reynolds, 1963b) more pronounced with lower constant rates of reinforcement. Thus, the upper function in Fig. 14 is shallower than the lower. In fact, as the reinforcement rate during the timeout increases, the proportion between the rates of responding in the two components should, according to equations 22 and 23, approach asymptotically the ratio between the rates of reinforcement, in this instance 3:1. The ratios of responding are, moving to the right along the abscissa, 1.19:1, 1.6:1; 2.24:1, 2.54:1; 2.69:1. The greater susceptibility to contrast of less frequently reinforced behavior has been noted by Bernheim and Williams (1967), as well as in various experiments on pigeons in multiple schedules. Their finding, based as it was on rats in running wheels, serves particularly to extend the applicability of the present analysis.

Although it is premature to argue for the exact function by which the interaction between components is diminished, the simple multiplicative factor of equation 20 does well with most of the published literature. Parameters have been calculated for three other multiple schedules in which reinforcement varied over a range large enough to allow some assessment. The fit of the data to theory was no worse than in Fig. 14, with no deviation greater than six responses per minute, and 15 (out of 18 independent data points) less than three responses per minute. Table 1 shows the parameter values for the three experiments, 
all of which used pigeons as subjects. Lander and Irwin's (1968) was a conventional twocomponent multiple schedule. Nevin's (1968) had as its independent variable the duration of reinforced non-responding in one of its two components. The shorter the duration, the higher the rate of reinforcement. The parameters predict the rate of responding in the other component, which was a conventional variable interval. Rachlin and Baum's (1969) experiment used two keys, of which one always provided reinforcement on VI 3-min. The reinforcer was 4 -sec access to food. The light on the other key was extinguished except when a second VI 3-min programmer completed an interval, at which time it was lit until the next response collected the reinforcement. ${ }^{6}$ The amount of these reinforcements was varied 16-fold during the course of the experiment. Responding on the other key varied inversely with magnitude of reinforcement, as if amount and frequency of reinforcement could be traded off against each other in equation 20.

Equation 20 appears to run into trouble mainly in regard to transitory effects. For example, Terrace $(1966 a,-b)$ found a transient contrast effect going from a simple variableinterval schedule to the same variable interval alternating with extinction. Equation 20 does not predict any change in rate here, since the interaction term is zero before and after the change. Terrace's procedure differs from Reynolds', which goes from a multiple schedule

\footnotetext{
'Catania's findings (1963b) with just this procedure are plotted as x's on Fig. 11. It was noted before (see p. 257) that the $x$ 's are slightly above the predicted line, which is where they should be if $\mathrm{m}<1.0$; in fact, $\mathrm{m}<1.0$ ( $\mathrm{m}=0.45$ in the Rachlin-Baum study) because the alternating conditions of reinforcement and stimulation make this more properly a multiple schedule than a concurrent, notwithstanding the use of two response keys.
}

consisting of a pair of matched variable intervals to one consisting of the variable interval alternating with extinction and for which equation 20 predicts contrast. Terrace's contrast effect vanishes with mere exposure to the conditions or with repeated alternations between the variable interval in isolation and the multiple schedule consisting of the variable interval and extinction. On the other hand, Bloomfield (1967b), using Reynolds' paradigm and addressing himself specifically to the persistence of contrast, found the effect undiminished even with several alternations back and forth between mult VI VI and mult VI EXT. Equation 20, then, is apparently consistent with the long-term effects, but not with the temporary contrast in the Terrace paradigm.

Terrace $(1966 b)$ concluded that the temporary contrast effect is emotional, based on the aversiveness of extinction. He noted the oneto-one correspondence between contrast and peak shift in discrimination learning, and also that the peak shift is often taken as evidence for an inhibitory or aversive effect of nonreinforcement. Any procedure that produces a peak shift also produces contrast; any procedure that prevents or diminishes the peak shift, prevents or diminishes contrast, according to Terrace. Aversiveness as negative reinforcement solves the problem, for adding a negative quantity to the denominator of $k R_{A}$ / $\left(R_{A}+R_{o}\right)$ increases the value of the expression, given that it remains positive. And there is other evidence that extinction is temporarily aversive as, for example, summarized by Terrace in his defense of the view. There is also evidence for representing aversiveness as a negative quantity. Brethower and Reynolds (1962) used a mult VI VI with equal reinforcement schedules in the two components, and added punishment with electric shock to one

Table 1

\begin{tabular}{lcccc}
\hline & $\begin{array}{c}k \\
\text { (Responses } \\
\text { per Minute) }\end{array}$ & $m$ & $\begin{array}{c}R_{0} \\
\text { (Reinforcements } \\
\text { per Hour) }\end{array}$ & Procedure \\
\hline \hline $\begin{array}{c}\text { Lander and Irwin } \\
\text { (1968) }\end{array}$ & 77 & 0.2 & 4 & VI 3-min VI X-min \\
$\begin{array}{c}\text { Nevin (1968) } \\
\text { Rachlin and Baum } \\
\text { (1969) }\end{array}$ & 85 & 0.35 & 2 & VI 3-min DRO x \\
& 70 & 0.45 & 10 & $\begin{array}{c}\text { VI 3-min CRF } \\
\text { (varying amount of } \\
\text { reinf }\end{array}$ \\
\hline
\end{tabular}


of the components. They found a contrast effect in which magnitude was an increasing function of the shock intensity.

A corrollary of the foregoing is that equation 20 predicts a difference between the rate on a simple variable-interval schedule and the rate on a multiple schedule in which each component uses this same variable interval, as follows:

$$
\frac{\mathbf{k} \mathbf{R}_{\mathbf{A}}}{\mathbf{R}_{\mathbf{A}}+\mathbf{R}_{\mathbf{o}}} \geq \frac{\mathbf{k} \mathbf{R}_{\mathbf{A}}}{\mathbf{R}_{\mathbf{A}}+\mathbf{m} \mathbf{R}_{\mathbf{A}}+\mathbf{R}_{\mathbf{o}}}
$$

Only if $\mathrm{m}=0$ will the two expressions be equal; otherwise the variable interval in isolation will produce the higher rate. This surprising prediction appears, in fact, to be correct. In Bloomfield's experiment (1967b), responding on a variable interval alone was faster than responding on a multiple comprising two components of the same variable interval, although the experiment was not specifically designed for this comparison. A direct test of the inequality above (24) is in an unpublished study by Terrace. Four pigeons were trained initially with a simple VI l-min in the presence of a given stimulus. Then, when responding was judged to be stable, this stimulus alternated every $1.5 \mathrm{~min}$ with a different stimulus, but reinforcement was continued on VI 1-min. For each pigeon, and for at least several weeks of daily sessions, the rate of responding dropped during the stimulus common to the first and second procedures. The average decrement was about $30 \%$, which for these reinforcement rates implies $0.31<\mathrm{m}<0.36$ (setting $\mathrm{R}_{0}=0-10$ ). At this point, however, the discussion begins to go beyond the boundaries set by well established data.

\section{ENVOI}

Temporary changes in responding clearly require further study, as the foregoing section shows. There are other, even more short-term, changes in responding (e.g., Williams, 1965; Nevin and Shettleworth, 1966; Bernheim and Williams, 1967) in multiple schedules that must for now also remain on the boundary of the present formulation. Another boundary, touched on earlier, is the definition of response classes (see p. 251). The key pecking of pigeons, for example, is relatively unambiguous, but even its properties, as some experi- ments show (Herrnstein, 1958), can get entangled with the measurement of response strength. Nevertheless, within these boundaries, the present formulation is a quantitative law of effect that cuts across traditional distinctions between choice and sheer output of behavior, as well as between simultaneous and successive conditions of work. The territory circumscribed is sizeable, expandable, and susceptible to precise measurement.

\section{REFERENCES}

Baum, W. H. and Rachlin, H. C. Choice as time allocation. Journal of the Experimental Analysis of Behavior, 1969, 12, 861-874.

Bernheim, J. W. and Williams, D. R. Time-dependent contrast effects in a multiple schedule of food reinforcement. Journal of the Experimental Analysis of Behavior, 1967, 10, 243-249.

Bitterman, M. E. Phyletic differences in learning. American Psychologist, 1965, 20, 396-410.

Bloomfield, T. M. Behavioral contrast and relative reinforcement in two multiple schedules. Journal of the Experimental Analysis of Behavior, 1967, 10, 151-158. (a)

Bloomfield, T. M. Some temporal properties of behavioral contrast. Journal of the Experimental Analysis of Behavior, 1967, 10, 159-164. (b)

Boneau, C. A. and Axelrod, S. Work decrement and reminiscence in pigeon operant responding. Journal of Experimental Psychology, 1962, 64, 352-354.

Brethower, D. M. and Reynolds, G. S. A facilitative effect of punishment on unpunished behavior. Journal of the Experimental Analysis of Behavior, 1962, 5, 191-199.

Brownstein, A. J. and Pliskoff, S. S. Some effects of relative reinforcement rate and changeover delay in response-independent concurrent schedules of reinforcement. Journal of the Experimental Analysis of Behavior, 1968, 11, 683-688.

Brunswik, E. Representative design and probabilistic theory in a functional psychology. Psychological Review, 1955, 62, 193-217.

Bush, R. R. and Mosteller, F. Stochastic models for learning. New York: Wiley, 1955.

Catania, A. C. Concurrent performances: a baseline for the study of reinforcement magnitude. Journal of the Experimental Analysis of Behavior, 1963, 6, 299-300. (a)

Catania, A. C. Concurrent performances: reinforcement interaction and response independence. Journal of the Experimental Analysis of Behavior, 1963, 6, 253-263. (b)

Catania, A. C. Concurrent operants. In W. K. Honig (Ed.), Operant behavior: areas of research and application. New York: Appleton-Century-Crofts, 1966. Pp. 213-270.

Catania, A. C. and Reynolds, G. S. A quantitative analysis of the responding maintained by interval schedules of reinforcement. Journal of the Experimental Analysis of Behavior, 1968, 11, 327-383.

Chung, S.-H. Some quantitative laws of operant be- 
havior. Unpublished doctoral dissertation, Harvard University, 1966.

Chung, S.-H. and Herrnstein, R. J. Choice and delay of reinforcement. Journal of the Experimental Analysis of Behavior, 1967, 10, 67-74.

Ferster, C. B. and Skinner, B. F. Schedules of reinforcement. New York: Appleton-Century-Crofts, 1957.

Findley, J. D. Preference and switching under concurrent scheduling. Journal of the Experimental Analysis of Behavior, 1958, 1, 123-144.

Guthrie, E. R. and Horton, G. P. Cats in a puzzle box. New York: Rinehart, 1946.

Herrnstein, R. J. Some factors influencing behavior in a two-response situation. Transactions of the New York Academy of Sciences, 1958, 21, 35-45.

Herrnstein, R. J. Relative and absolute strength of response as a function of frequency of reinforcement. Journal of the Experimental Analysis of Behavior, 1961, 4, 267-272.

Herrnstein, R. J. Secondary reinforcement and rate of primary reinforcement. Journal of the Experimental Analysis of Behavior, 1964, 7, 27-36.

Herrnstein, R. J. and Morse, W. H. A conjunctive schedule of reinforcement. Journal of the Experimental Analysis of Behavior, 1958, 1, 15-24.

Holz, W. C. Punishment and rate of positive reinforcement. Journal of the Experimental Analysis of Behavior, 1968, 11, 285-292.

Hull, C. L. Principles of behavior. New York: Appleton-Century, 1943.

Lander, D. G. and Irwin, R. J. Multiple schedules: effects of the distribution of reinforcements between components on the distribution of responses between components. Journal of the Experimental Analysis of Behavior, 1968, 11, 517-524.

Morse, W. H. Intermittent reinforcement. In W. $\mathbf{K}$. Honig (Ed.), Operant behavior: areas of research and application. New York: Appleton-CenturyCrofts, 1966. Pp. 52-108.

Natapoff, A. How symmetry restricts symmetric choice. Journal of Mathematical Psychology, in press.

Neuringer, A. J. Choice and rate of responding in the pigeon. Unpublished doctoral dissertation, Harvard University, 1967. (a)

Neuringer, A. J. Effects of reinforcement magnitude on choice and rate of responding. Journal of the Experimental Analysis of Behavior, 1967, 10, 417424. (b)

Nevin, J. A. Differential reinforcement and stimulus control of not responding. Journal of the Experimental Analysis of Behavior, 1968, 11, 715-726.

Nevin, J. A. Signal detection theory and operant behavior. Review of D. M. Green and J. A. Swets' Signal detection theory and psychophysics. Journal of the Experimental Analysis of Behavior, 1969, 12, 475-480.

Nevin, J. A. and Shettleworth, S. J. An analysis of contrast effects in multiple schedules. Journal of the Experimental Analysis of Behavior, 1966, 9, 305-315.

Norman, M. F. An approach to free-responding on schedules that prescribe reinforcement probability as a function of interresponse time. Journal of Mathematical Psychology, 1966, 3, 235-268.

Pliskoff, S. S. Rate-change effects with equal potential reinforcements during the "warning" stimulus. Journal of the Experimental Analysis of Behavior, $1963,6,557-562$.

Rachlin, H. and Baum, W. M. Response rate as a function of amount of reinforcement for a signalled concurrent response. Journal of the Experimental Analysis of Behavior, 1969, 12, 11-16.

Reynolds, G. S. An analysis of interactions in a multiple schedule. Journal of the Experimental Analysis of Behavior, 1961, 4, 107-117. (a)

Reynolds, G. S. Behavioral contrast. Journal of the Experimental Analysis of Behavior, 1961, 4, 57-71. (b)

Reynolds, G. S. Relativity of response rate and reinforcement frequency in a multiple schedule. Journal of the Experimental Analysis of Behavior, 1961, 4, 179-184. (c)

Reynolds, G. S. On some determinants of choice in pigeons. Journal of the Experimental Analysis of Behavior, 1963, 6, 53-59. (a)

Reynolds, G. S. Some limitations on behavioral contrast and induction during successive discrimination. Journal of the Experimental Analysis of Behavior, 1963, 6, 131-139. (b)

Shimp, C. P. Probablistically reinforced choice behavior in pigeons. Journal of the Experimental Analysis of Behavior, 1966, 9, 443-455.

Shull, R. L. and Pliskoff, S. S. Changeover delay and concurrent performances: some effects on relative performance measures. Journal of the Experimental Analysis of Behavior, 1967, 10, 517-527.

Skinner, B. F. The behavior of organisms. New York: Appleton-Century, 1938.

Skinner, B. F. The nature of the operant reserve. Psychological Bulletin, 1940, 37, 423 (abstract).

Skinner, B. F. Science and human behavior. New York: Macmillan, 1953.

Terrace, H. S. Behavioral contrast and the peak shift: effects of extended discrimination training. Journal of the Experimental Analysis of Behavior, 1966, 9, 613-617. (a)

Terrace, H. S. Stimulus control. In W. K. Honig (Ed.), Operant behavior: areas of research and application. New York: Appleton-Century-Crofts, 1966. Pp. 271344. (b)

Thorndike, E. L. Animal intelligence. New York: Macmillan, 1911.

Tolman, E. C. The determiners of behavior at a choice point. Psychological Review, 1938, 45, 1-41.

Tolman, E. C. Cognitive maps in rats and men. Psychological Review, 1948, 55, 189-208.

Williams, D. R. Negative induction in instrumental behavior reinforced by central stimulation. Psychonomic Science, 1965, 2, 341-342.

Received 3 December 1969. 